\title{
QUALITY OF GROUND WATER IN HARRISON COUNTY, MISSISSIPPI, JUNE - JULY 1993
}

By Larry J. Slack, William T. Oakley, Charles G. O'Hara, and Lance M. Cooper U.S. GEOLOGICAL SURVEY Open-File Report 94-125

Prepared in cooperation with the HARRISON COUNTY BOARD OF SUPERVISORS

Jackson, Mississippi

1994 


\section{U.S. DEPARTMENT OF THE INTERIOR}

BRUCE BABBITT, Secretary

U.S. GEOLOGICAL SURVEY

Gordon P. Eaton, Director

For additional information write to:

District Chief

U.S. Geological Survey

Suite 710, Federal Building $100 \mathrm{~W}$. Capitol Street

Jackson, Mississippi 39269
Copies of this report can be purchased from:

U.S. Geological Survey

Earth Science Information Center

Open-File Reports Section

Box 25286, MS 517, Federal Center

Denver, Colorado 80225 


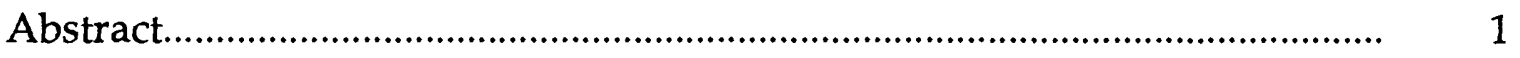

Introduction ........................................................................................... 2

Site selection criteria and site information ..................................................... 2

Methods of sampling and analysis................................................................. 3

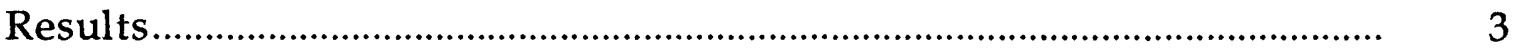

Summary ..................................................................................................

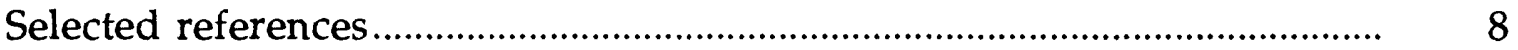

\section{ILLUSTRATIONS}

Figure 1. Map showing location of ground-water sites in Harrison County, Mississippi, with water-quality analyses during 1993............ 9

Figures 2-6. Maps of Harrison County, Mississippi, showing:

2. Specific conductance of ground water during 1993 ......................... 10

3. $\mathrm{pH}$ of ground water during 1993........................................................... 11

4. Color of ground water during 1993 ............................................ 12

5. Dissolved-solids concentration in ground water during 1993...... 13

6. Chloride concentration in ground water during 1993................... 14

Figures 7-11. Graphs for Harrison County, Mississippi, showing relation of depth and:

7. Specific conductance of ground water during 1993 .......................... 15

8. $\mathrm{pH}$ of ground water during 1993........................................................ 16

9. Color of ground water during 1993 ................................................. 17

10. Dissolved-solids concentration in ground water during 1993...... 18

11. Chloride concentration in ground water during 1993................... 19 


\section{TABLES}

Page

Table 1. Summary of site information for wells in Harrison County,

Mississippi.

2. Water-quality data collected from wells in Harrison County,

Mississippi, during 1993

3. Water-quality data collected from selected wells in Harrison County, Mississippi, during 1993.

4. Summary of water-quality data collected from wells in Harrison County, Mississippi, during 1993

\section{CONVERSION FACTORS AND WATER-QUALITY INFORMATION}

Multiply

foot
By

0.3048 $\underline{\text { To obtain }}$

meter

To convert degrees Celsius $\left({ }^{\circ} \mathrm{C}\right)$ to Fahrenheit $\left({ }^{\circ} \mathrm{F}\right)$, use the following:

$$
{ }^{\circ} \mathrm{C}=5 / 9\left({ }^{\circ} \mathrm{F}-32\right)
$$

Specific conductance is reported in microsiemens per centimeter at $25^{\circ} \mathrm{C}$ $(\mu \mathrm{S} / \mathrm{cm}) ; \mathrm{pH}$, in standard units. Chemical concentrations are given in milligrams per liter $(\mathrm{mg} / \mathrm{L})$ or micrograms per liter $(\mu \mathrm{g} / \mathrm{L})$. Milligrams and micrograms per liter are units expressing the weight of solute per volume (liter) of water. One thousand micrograms per liter is equivalent to $1 \mathrm{mg} / \mathrm{L}$. For concentrations less than $7,000 \mathrm{mg} / \mathrm{L}$, milligrams per liter are equivalent to parts per million. Color is reported in platinum-cobalt units. 


\section{QUALITY OF GROUND WATER IN HARRISON COUNTY, MISSISSIPPI, JUNE - JULY 1993}

by Larry J. Slack, William T. Oakley, Charles G. O'Hara, and Lance M. Cooper

ABSTRACT

During June and July 1993, the U.S. Geological Survey analyzed water from 145 wells in Harrison County, southeastern Mississippi. The wells are completed in five major geologic units: the Citronelle, Graham Ferry, Pascagoula, and Hattiesburg Formations and the Catahoula Sandstone. The wells ranged from 74 to 2,410 feet in depth. Specific conductance (lab), ranged from 15 to 2,020 microsiemens per centimeter; $\mathrm{pH}$ (lab), from 5.9 to 9.0; color, from less than 5 to 120 platinum-cobalt units; dissolved-solids concentrations (residue on evaporation), from 20 to 1,120 milligrams per liter; chloride concentrations, from 1.9 to $\mathbf{4 7 0}$ milligrams per liter; and nitrite plus nitrate as nitrogen concentrations, from less than $\mathbf{0 . 0 2}$ to $\mathbf{0 . 8 5}$ milligram per liter. Most of the larger values of specific conductance, $\mathrm{pH}$, dissolvedsolids concentrations, and chloride concentrations were from wells in the southern one-half of the county. 


\section{INTRODUCTION}

As part of the Federal-State Cooperative Program, the U.S. Geological Survey (USGS) collects, on a systematic basis, data needed for the continuing determination and evaluation of the quantity, quality, and use of water resources in the United States and appraises the availability of ground and surface water through analytical and interpretive investigations. The resulting information forms the foundation for many of the nation's waterresources management and planning activities and allows for the detection of water problems.

During June and July 1993, in cooperation with the Harrison County Board of Supervisors, the USGS analyzed water from 145 wells to determine current water quality of the ground water in the southeastern Mississippi county. This report summarizes the site selection criteria and site information, the methods of sampling and analysis, and the results of the water-quality data for these wells.

\section{SITE SELECTION CRITERIA AND SITE INFORMATION}

Sites were selected to represent the major geologic units and depths at which wells have been completed in Harrison County. First preference in site selection was given to wells included in the USGS ground-water sampling network in the county in 1988. Other selection criteria included wells that were currently (1993) in use or had been in use recently.

Two of the 145 wells are completed in the Citronelle Formation, 55 in the Graham Ferry Formation, 72 in the Pascagoula Formation, 14 in the Hattiesburg Formation, and 2 in the Catahoula Sandstone (table 1, fig. 1). Water from 64 wells (44 percent) is used for public supply; 54 wells (37 percent) for domestic purposes; 13 wells (9 percent) for industrial purposes; and 7 wells ( 5 percent) for institutional purposes. Water from the remainder of the wells is used for aquaculture, fire protection, irrigation, power generation, or recreation purposes. 


\section{METHODS OF SAMPLING AND ANALYSIS}

To obtain samples that closely represent ground water from the waterbearing unit, most of the wells were pumped long enough prior to sampling to withdraw at least twice the volume of water standing in the casing. The samples were shipped to the USGS Water Resources Division Water-Quality Service Unit in Ocala, Florida, where the samples were analyzed using standard USGS procedures.

\section{RESULTS}

Water-quality data collected from the 145 wells during the 1993 sampling are listed in table 2. These data include specific conductance (field and lab), $\mathrm{pH}$ (field and lab), color, dissolved-solids concentration (residue on evaporation at $180{ }^{\circ} \mathrm{C}$ ), chloride, and nitrite plus nitrate-nitrogen concentrations. The values for these constituents or properties are similar to those reported by Slack and othèrs (1993) for ground-water samples collected from March to June 1993 in Jackson County, immediately east of Harrison County.

Additional water-quality data collected from 27 wells selected from the the 145 wells are listed in table 3 . These data include nutrients (ammonia and orthophosphorus), major ions (calcium, magnesium, sodium, potassium, sulfate, fluoride, and silica), iron, manganese, and alkalinity.

For the convenience of the reader, the water-quality data collected from the 145 wells in Harrison County during the 1993 sampling are summarized in table 4. The data are presented individually for each of the major geologic units and then aggregated for all geologic units.

Also for the convenience of the reader, the areal distribution of specific conductance (lab), pH (lab), color, dissolved-solids concentration, and chloride concentration in ground water is mapped in figures 2-6. The relation of depth and specific conductance (lab), $\mathrm{pH}$ (lab), color, dissolved-solids concentration, and chloride concentration in ground water is shown in figures 7-11. 
The wells ranged from 74 to 2,410 feet in depth and had a median depth of 746 feet (table 4). Well depths generally were smallest for those completed in the Citronelle Formation and greatest for wells completed in the Catahoula Sandstone. Median depth was 115 feet for wells completed in the Citronelle Formation; 420 feet for wells completed in the Graham Ferry Formation; 842 feet for wells completed in the Pascagoula Formation; 1,675 feet for wells completed in the Hattiesburg Formation; and 2,405 feet for wells completed in the Catahoula Sandstone (table 4).

Specific conductance (lab) ranged from 15 to 2,020 microsiemens per centimeter; the median was 273 microsiemens (table 4). Most of the larger values of specific conductance (exceeding 500 microsiemens) were from wells in the southern one-half of the county (fig. 2). Specific conductance generally increased with depth in water from the Graham Ferry and Pascagoula Formations; however, the relation showed considerable variation (fig. 7).

The $\mathrm{pH}$ (lab) ranged from 5.9 to 9.0; the median was 8.4 (table 4). Most of the larger values of $\mathrm{pH}$ (exceeding 8.0 units) were from wells in the southern one-half of the county (fig. 3). The pH generally increased with depth in water from the Graham Ferry and Pascagoula Formations and decreased with depth in water from the Hattiesburg Formation; however, the relation showed considerable variation (fig. 8).

Color ranged from less than 5 to 120 platinum-cobalt units; the median was less than 5 platinum-cobalt units (table 4). Color was less than 5 platinum-cobalt units in two-thirds of the samples. Color exceeded 15 platinum-cobalt units in only 14 of 145 samples (less than 10 percent). Furthermore, color exceeded 75 units in only one sample (less than 1 percent). Most of the larger values of color (exceeding 35 units) were from wells in the northern one-half of the county (fig. 4). Color generally increased with depth in water from the Pascagoula Formation and decreased with depth in water from the Graham Ferry and Hattiesburg Formations; however, the relation showed considerable variation (fig. 9). 
The determination of color represents an evaluation of a physical property. The color of natural water usually results from leaching of organic debris (Hem, 1985). The recommended limit of 15 platinum-cobalt units for color in public water supplies is based principally on aesthetic reasons (U.S. Environmental Protection Agency, 1986). Water consistently can be treated using standard coagulation, sedimentation, and filtration processes to decrease color to substantially less than 15 platinum-cobalt units when the source water does not exceed 75 platinum-cobalt units.

Dissolved-solids concentrations (residue on evaporation) ranged from 20 to 1,120 milligrams per liter; the median was 199 milligrams per liter (table 4). Dissolved solids exceeded 500 milligrams per liter in only 6 of 145 samples (4 percent). Furthermore, dissolved solids exceeded 1,000 milligrams per liter in only two samples (about 1 percent). Most of the larger values of dissolved-solids concentrations (exceeding 250 milligrams per liter) were from wells in the southern one-half of the county (fig. 5). Dissolved-solids concentrations generally increased with depth in water from the Graham Ferry and Pascagoula Formations; however, the relation showed considerable variation (fig. 10).

High dissolved-solids concentrations are objectionable because of possible physiological effects, mineral taste, and economic consequences of additional water treatment. The recommended limit of 500 milligrams per liter for dissolved solids in public water supplies is based largely on aesthetic reasons (U.S. Environmental Protection Agency, 1986). One concern is that drinking water containing a high concentration of dissolved solids is likely to contain an excessive concentration of some specific substance that would be objectionable. Nationally, a considerable number of water supplies with dissolved solids in excess of the 500-milligram per liter limit are used without any obvious ill effects. On the basis of practicality, a dissolved-solids concentration of 1,000 milligrams per liter generally is recommended as an upper limit for potable waters. 
Chloride concentrations ranged from 1.9 to 470 milligrams per liter; the median was 3.7 milligrams per liter (table 4). Chloride concentrations exceeded 250 milligrams per liter in only 3 of 145 samples (about 2 percent) and did not exceed 500 milligrams per liter in any samples. Most of the larger values of chloride concentrations (exceeding 25 milligrams per liter) were from wells in the southern one-half of the county (fig. 6). Chloride concentrations generally increased with depth in water from the Graham Ferry and Pascagoula Formations; however, the relation showed considerable variation (fig. 11).

The recommended limit of 250 milligrams per liter for chloride in public water supplies is based largely on aesthetic reasons (U.S. Environmental Protection Agency, 1986). On the basis of taste, there may be a large difference between detectable and objectionable chloride concentrations; acclimatization is thought to be an important factor.

Nitrite plus nitrate as nitrogen concentrations ranged from less than 0.02 to 0.85 milligram per liter; the median was less than 0.02 milligram per liter (table 4). Nitrite plus nitrate as nitrogen concentrations were 0.14 milligram per liter or less in 141 of 143 samples (almost 99 percent). The primary maximum contaminant level for nitrate-nitrogen is 10 milligrams per liter (U.S. Environmental Protection Agency, 1986). Nitrite plus nitrate as nitrogen concentrations were small and showed no pattern of distribution areally or with depth. 


\section{SUMMARY}

During June and July 1993, the U.S. Geological Survey analyzed water from 145 wells in Harrison County, southeastern Mississippi. The wells are completed in five major geologic units: the Citronelle, Graham Ferry, Pascagoula, and Hattiesburg Formations and the Catahoula Sandstone.

The wells ranged from 74 to 2,410 feet in depth. Specific conductance (lab) ranged from 15 to 2,020 microsiemens per centimeter. The $\mathrm{pH}$ (lab) ranged from 5.9 to 9.0. Color ranged from less than 5 to 120 platinumcobalt units. Dissolved-solids concentrations (residue on evaporation) ranged from 20 to 1,120 milligrams per liter. Chloride concentrations ranged from 1.9 to 470 milligrams per liter. Nitrite plus nitrate as nitrogen concentrations ranged from less than 0.02 to 0.85 milligram per liter. Most of the larger values of specific conductance, $\mathrm{pH}$, dissolved-solids concentrations, and chloride concentrations were from wells in the southern one-half of the county. Most of the larger values of color were from wells in the northern one-half of the county. Specific conductance, $\mathrm{pH}$, color, dissolved-solids concentrations, and chloride concentrations showed considerable variation with depth. 


\section{SELECTED REFERENCES}

Brown, G.F., Foster, V.M., Adams, R.W., Reed, E.W., and Padgett, D.H., Jr., 1944, Geology and ground-water resources of the coastal area in Mississippi: Mississippi State Geological Survey Bulletin 60, 229 p.

Hem, J.D., 1985, Study and interpretation of the chemical characteristics of natural water (3d ed.): U.S. Geological Survey Water-Supply Paper 2254, $263 \mathrm{p}$.

Shattles, D.E., and Callahan, J.A., 1970, Water-level and water-quality trends in aquifers along the Mississippi Gulf Coast, 1970: Mississippi Board of Water Commissioners Bulletin 70-1, 25 p.

Slack, L.J., and Darden, Daphne, 1991, Summary of aquifer tests in Mississippi, June 1942 through May 1988: U.S. Geological Survey Water-Resources Investigations Report 90-4155, 40 p.

Slack, L.J., Oakley, W.T., and Cooper, L.M., 1993, Quality of ground water in Jackson County, Mississippi, March-June 1993: U.S. Geological Survey Open-File Report 93-479, 38 p.

U.S. Environmental Protection Agency, 1986, Quality criteria for water: U.S. Environmental Protection Agency, EPA--440/5-86-001, variously paginated.

Wasson, B.E., 1986, Sources for water supplies in Mississippi: Mississippi Research and Development Center Bulletin, 113 p. 


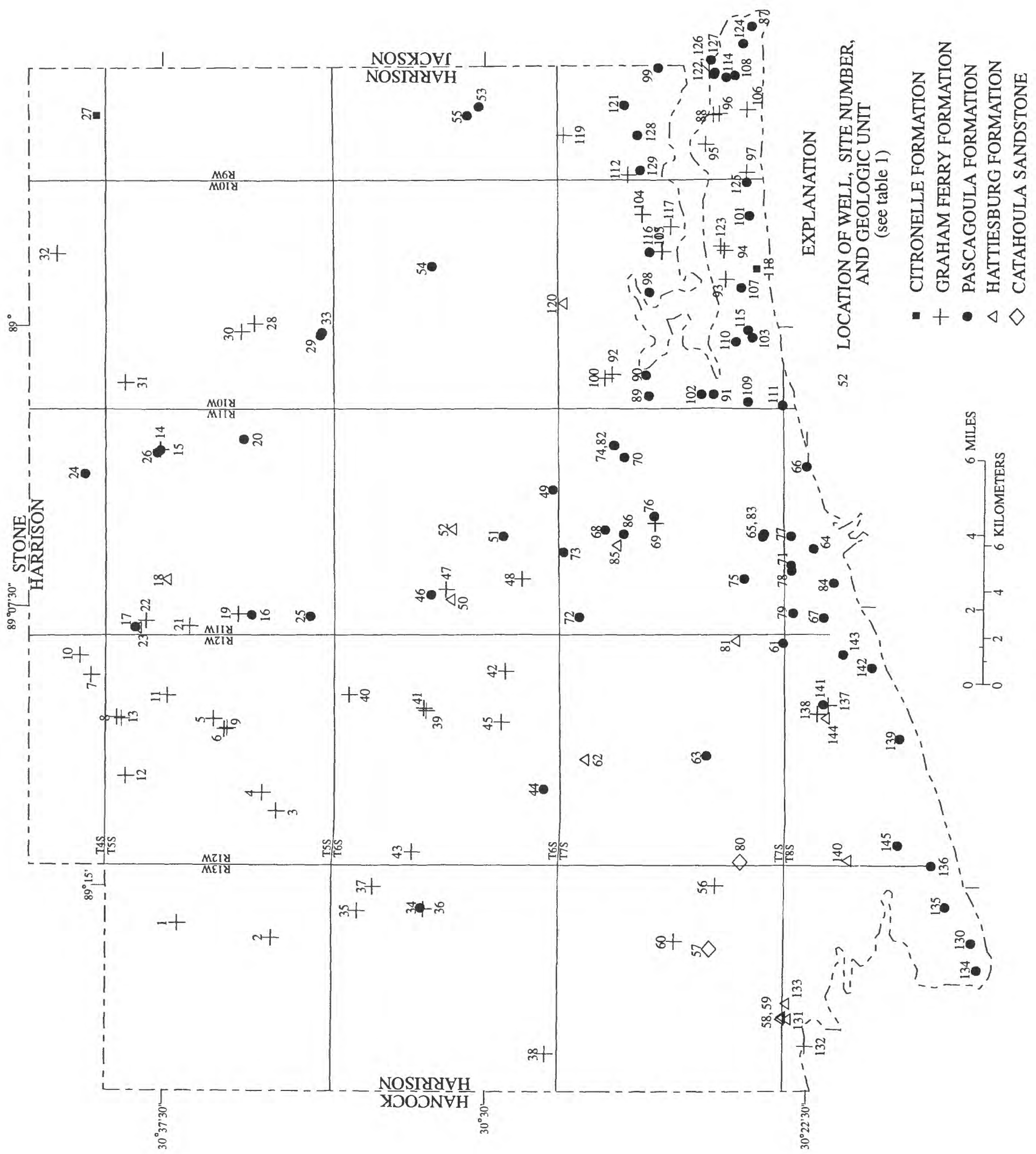

Figure 1. - Location of ground-water sites in Harrison County, Mississippi, with water-quality analyses during 1993. 


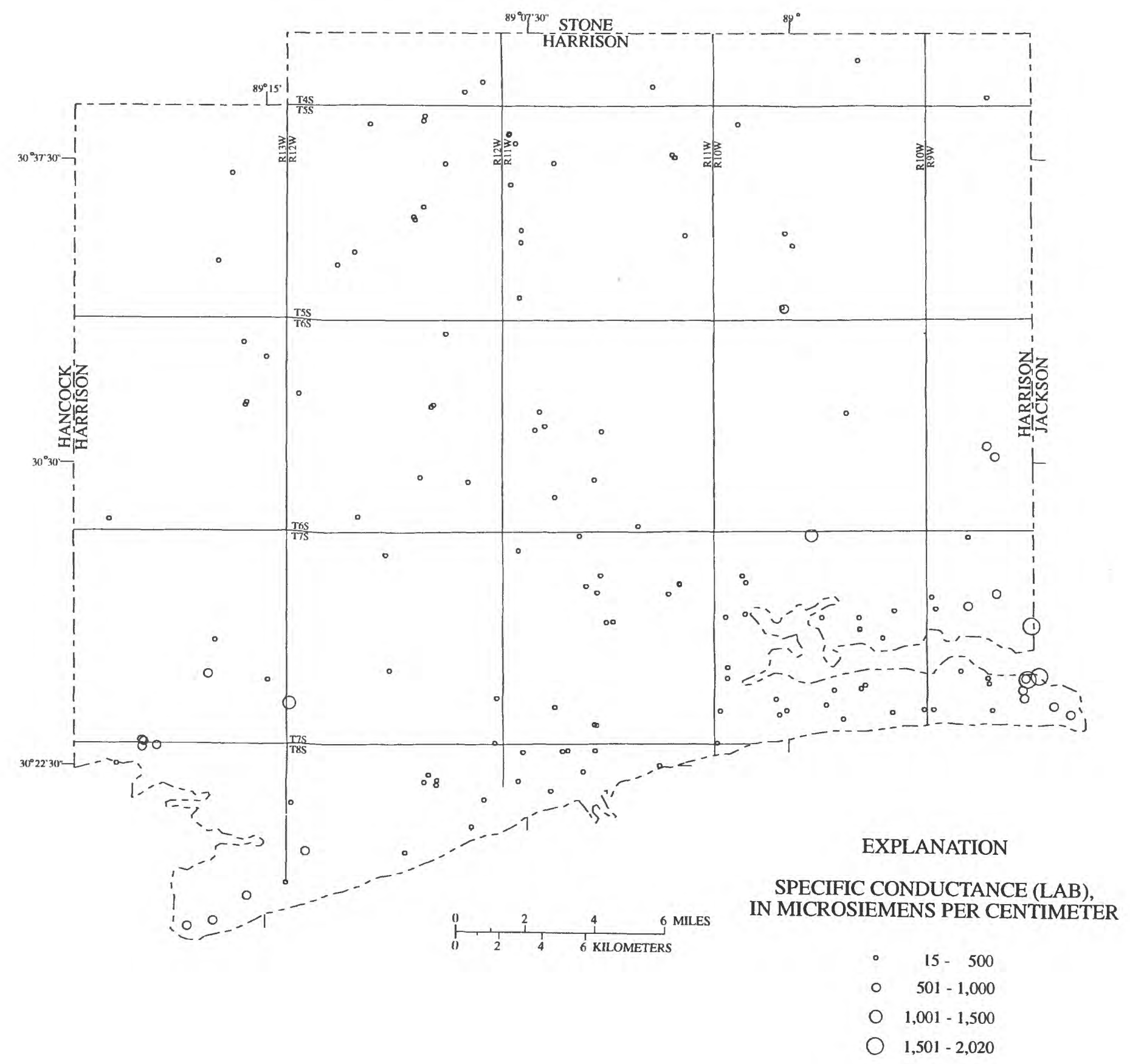

Figure 2.--Specific conductance (lab) of ground water in Harrison County, Mississippi, during 1993. 


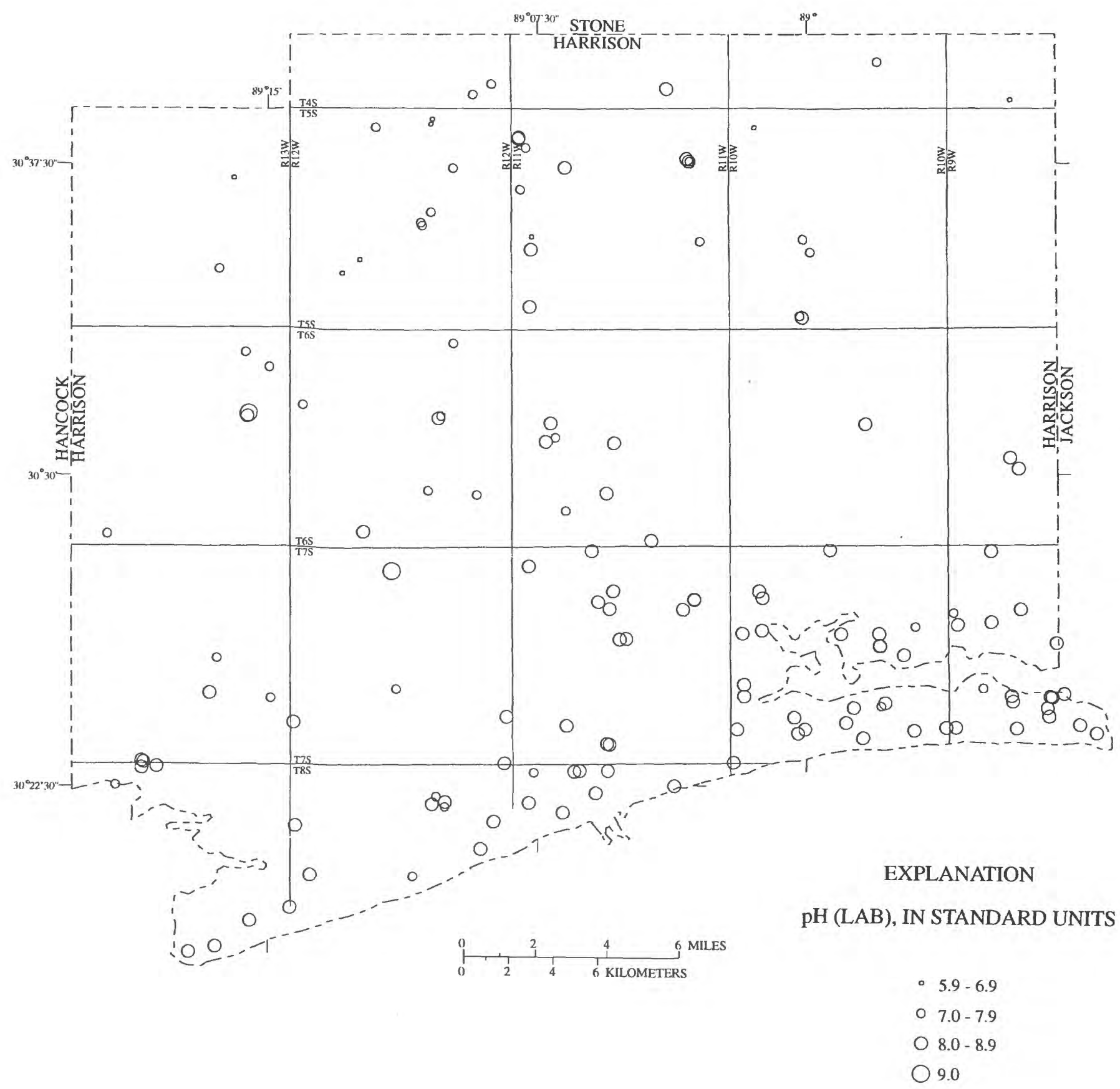

Figure 3.-pH (lab) of ground water in Harrison County, Mississippi, during 1993. 


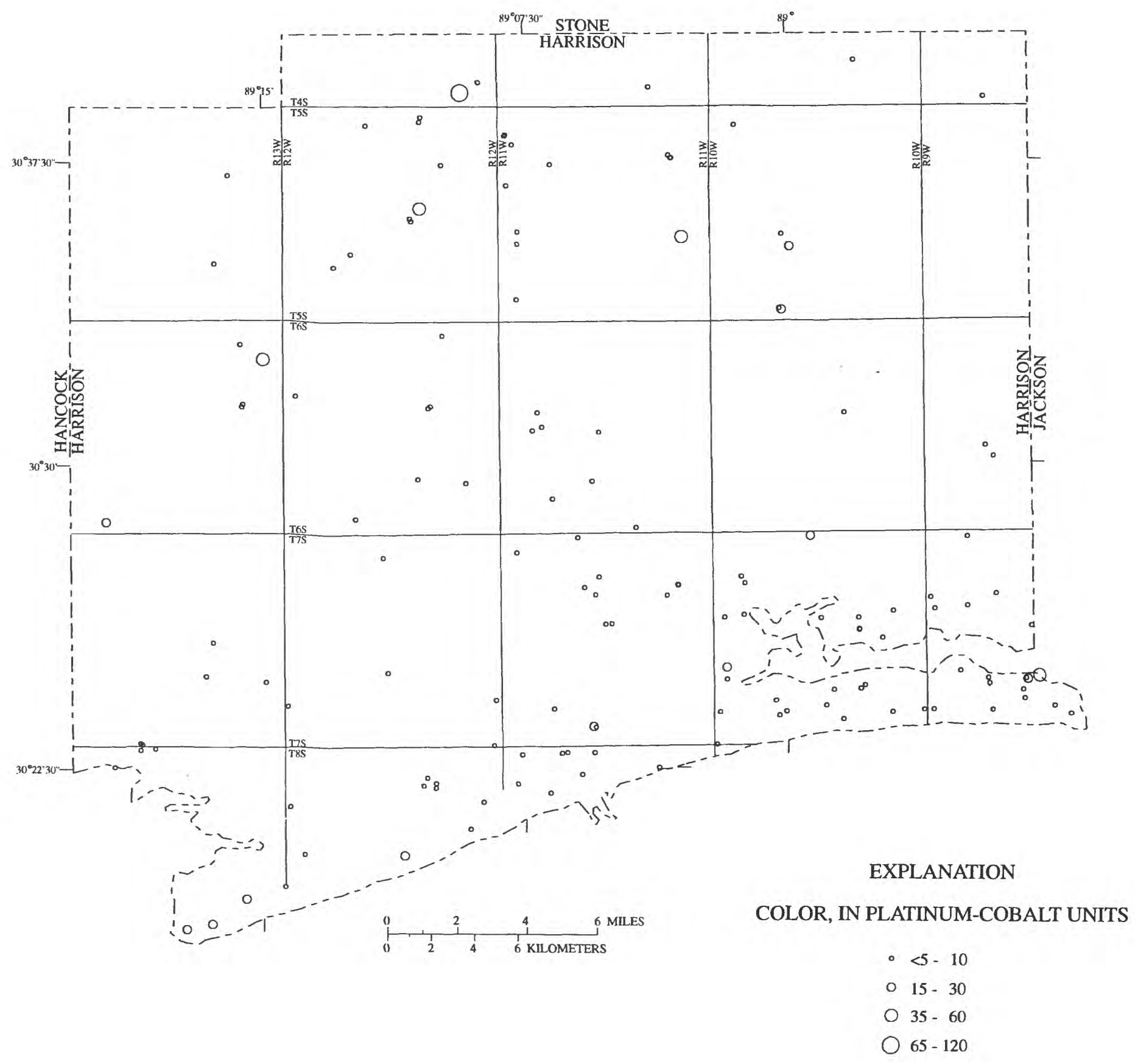

Figure 4.-Color of ground water in Harrison County, Mississippi, during 1993. 


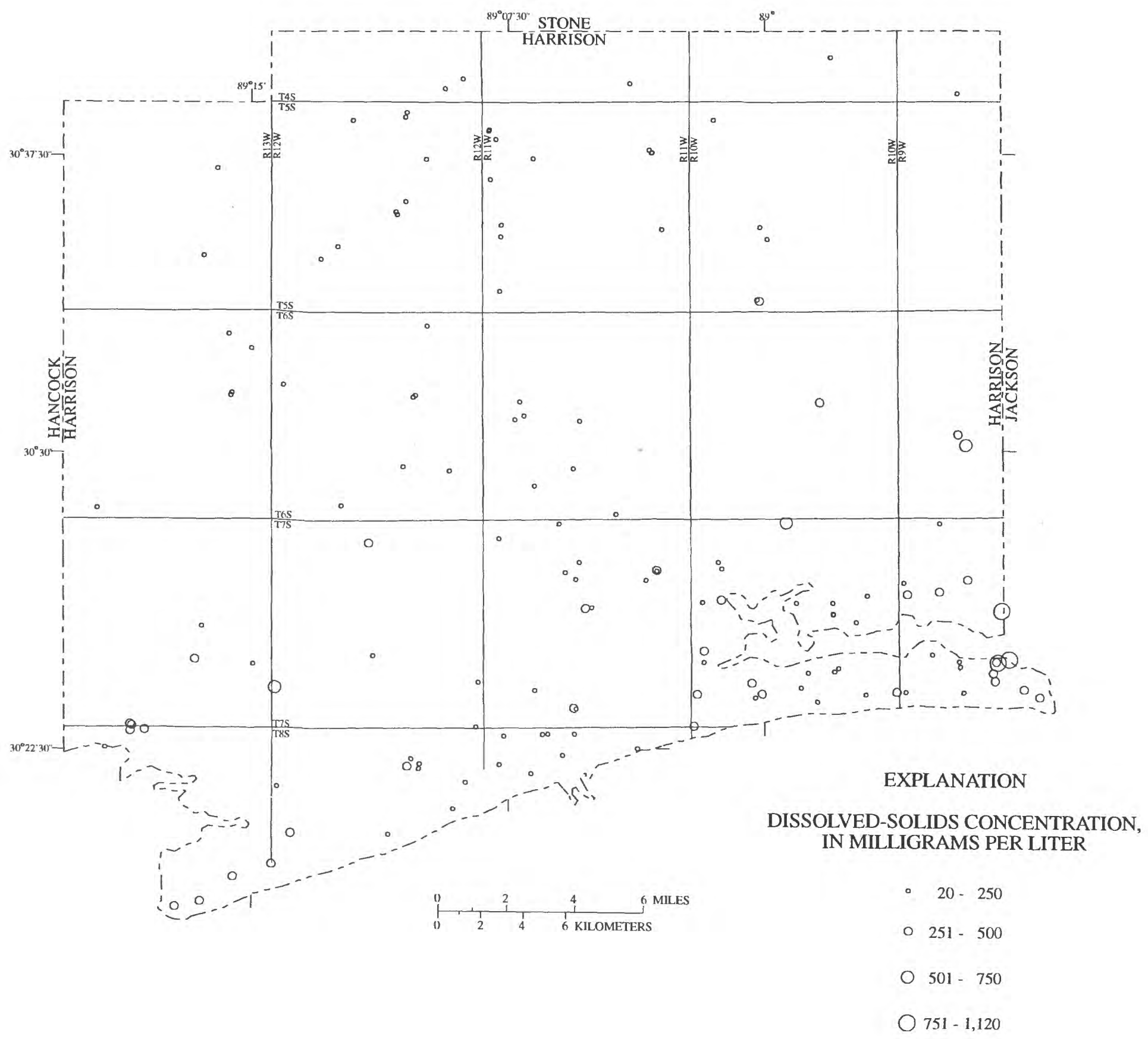

Figure 5.-Dissolved-solids concentration in ground water in Harrison County, Mississippi, during 1993. 


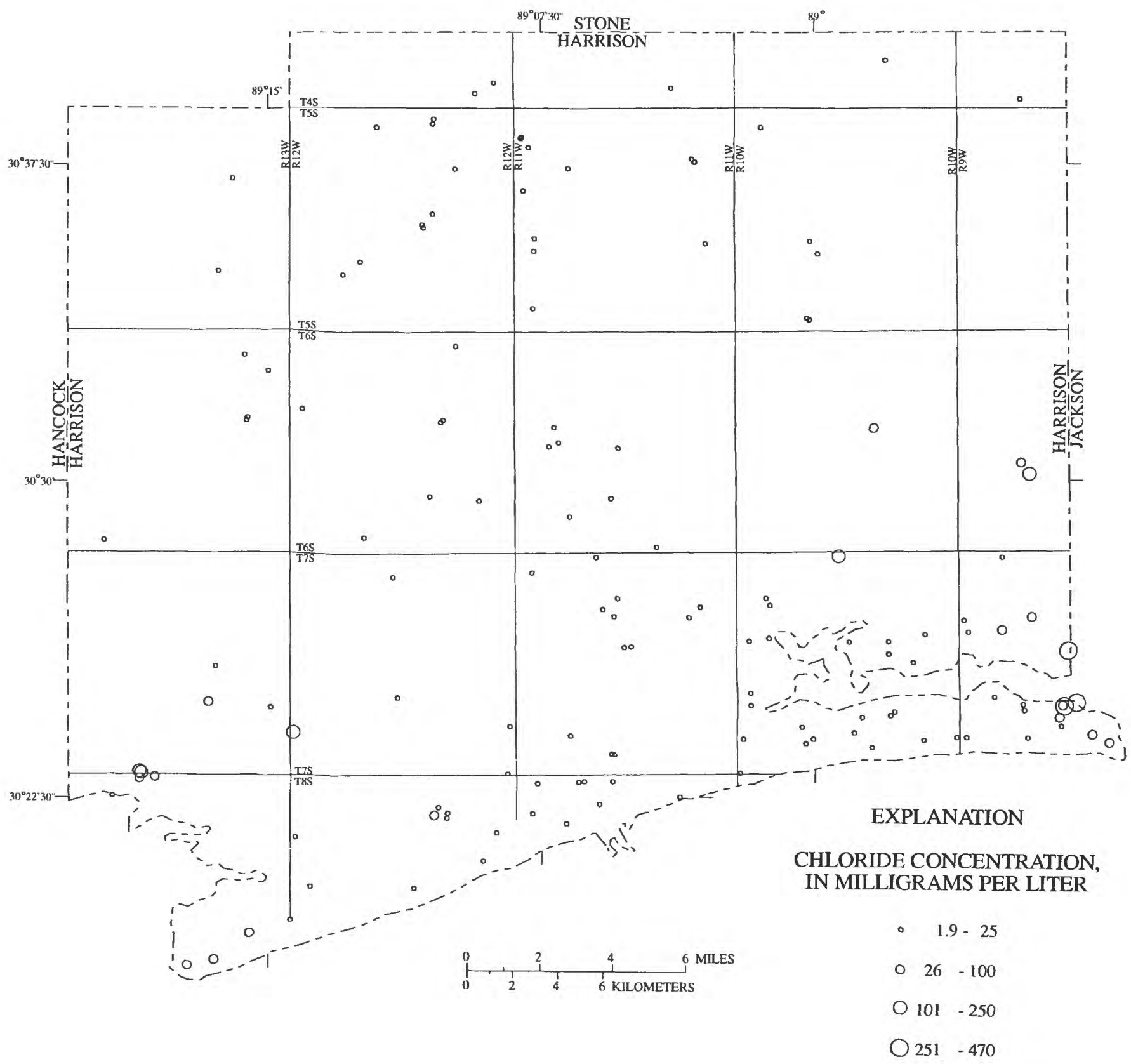

Figure 6.-Chloride concentration in ground water in Harrison County, Mississippi, during 1993. 


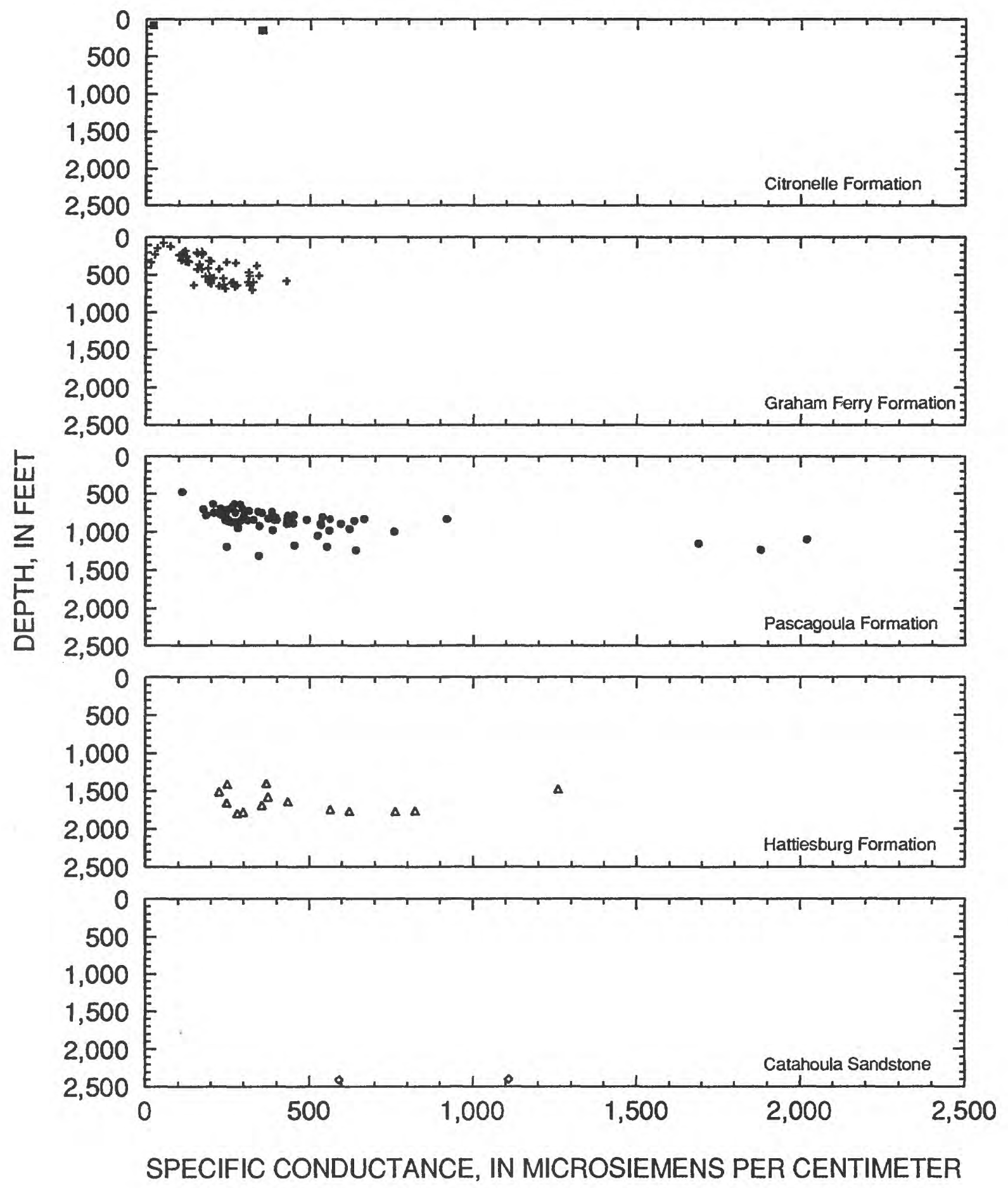

Figure 7.--Relation of depth and specific conductance of ground water in Harrison County, Mississippi, during 1993. 


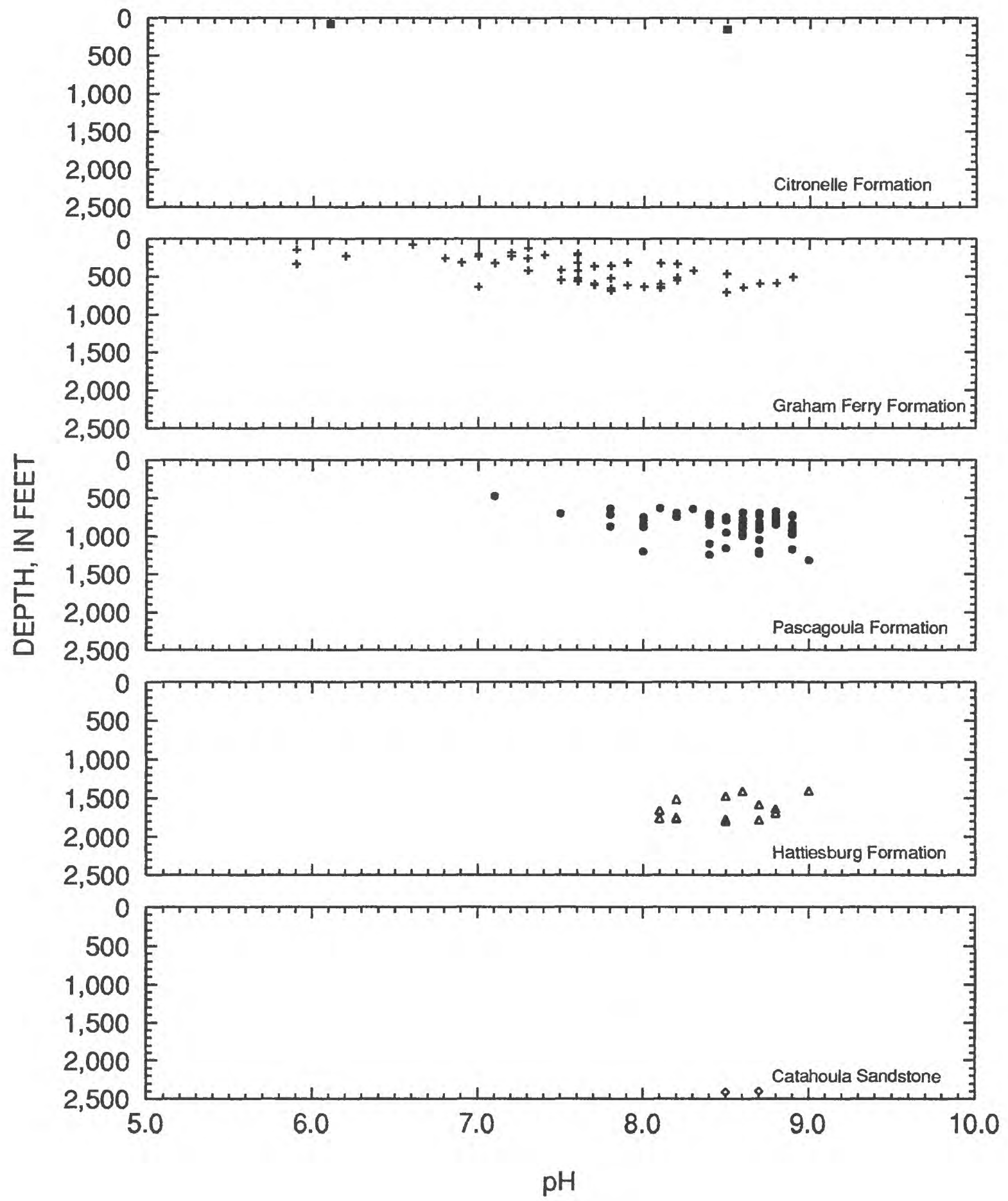

Figure 8.--Relation of depth and $\mathrm{pH}$ of ground water in Harrison County, Mississippi, during 1993. 


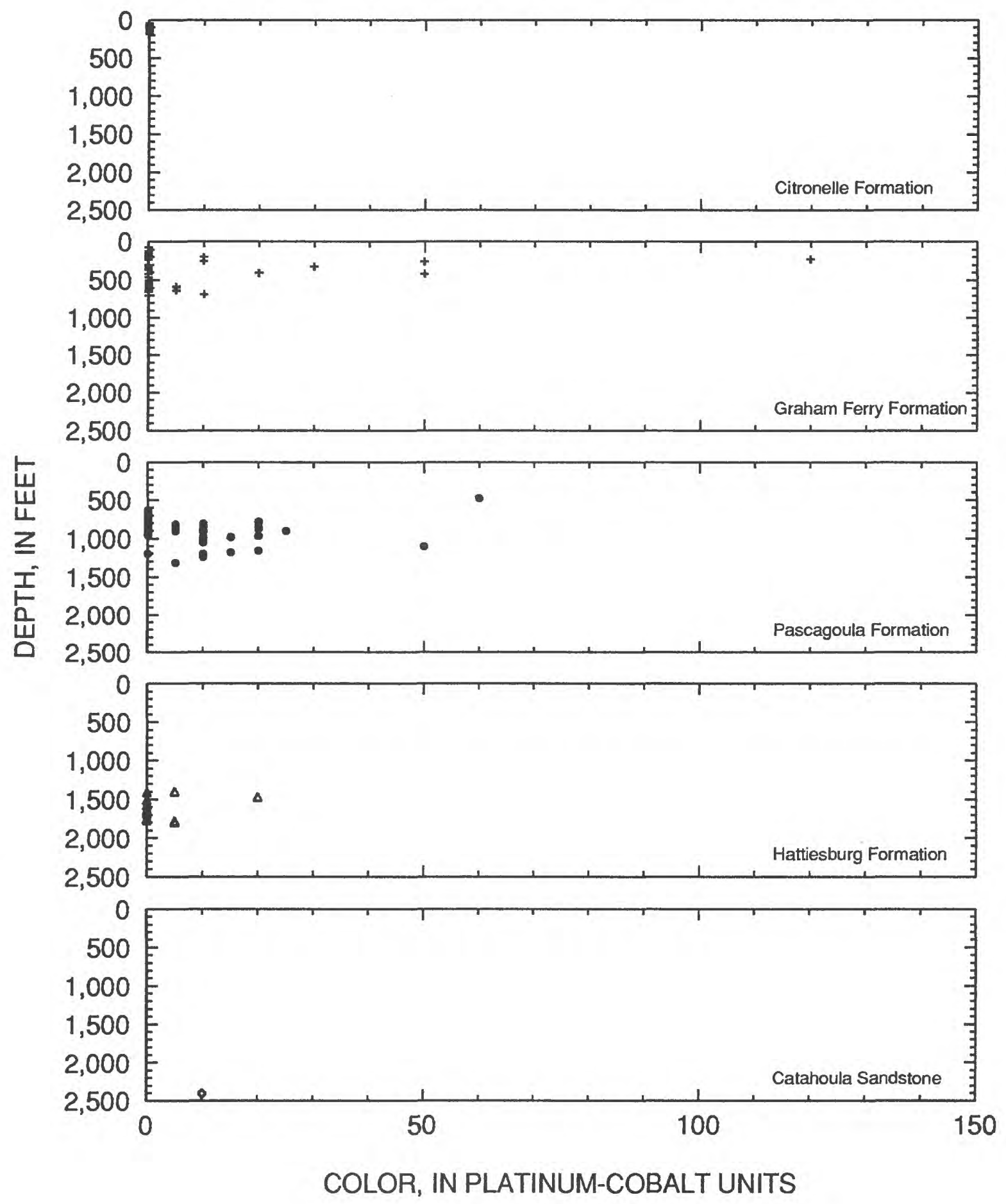

Figure 9.--Relation of depth and color of ground water in Harrison County, Mississippi, during 1993. 


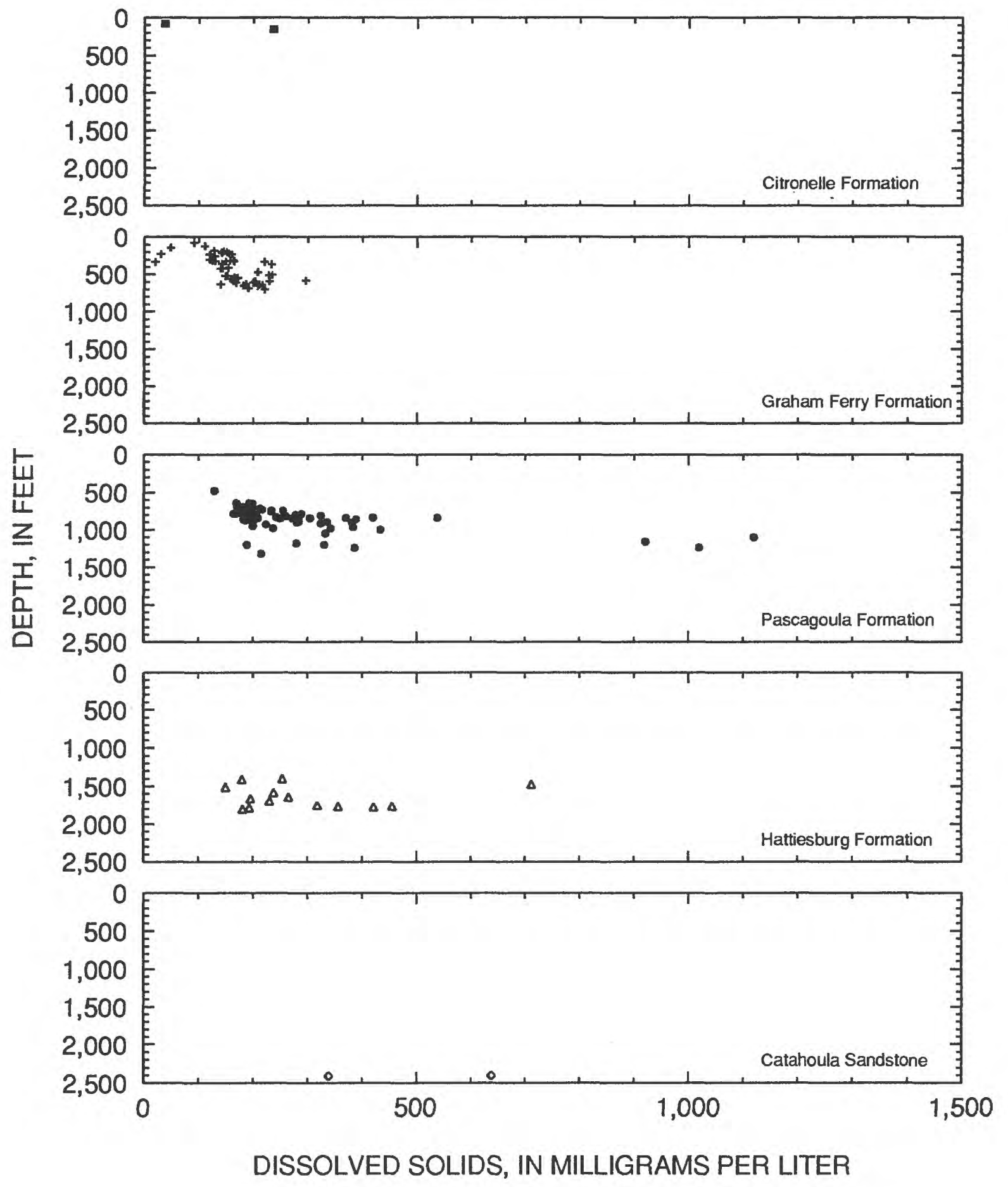

Figure 10.--Relation of depth and dissolved-solids concentration in ground water in Harrison County, Mississippi, during 1993. 


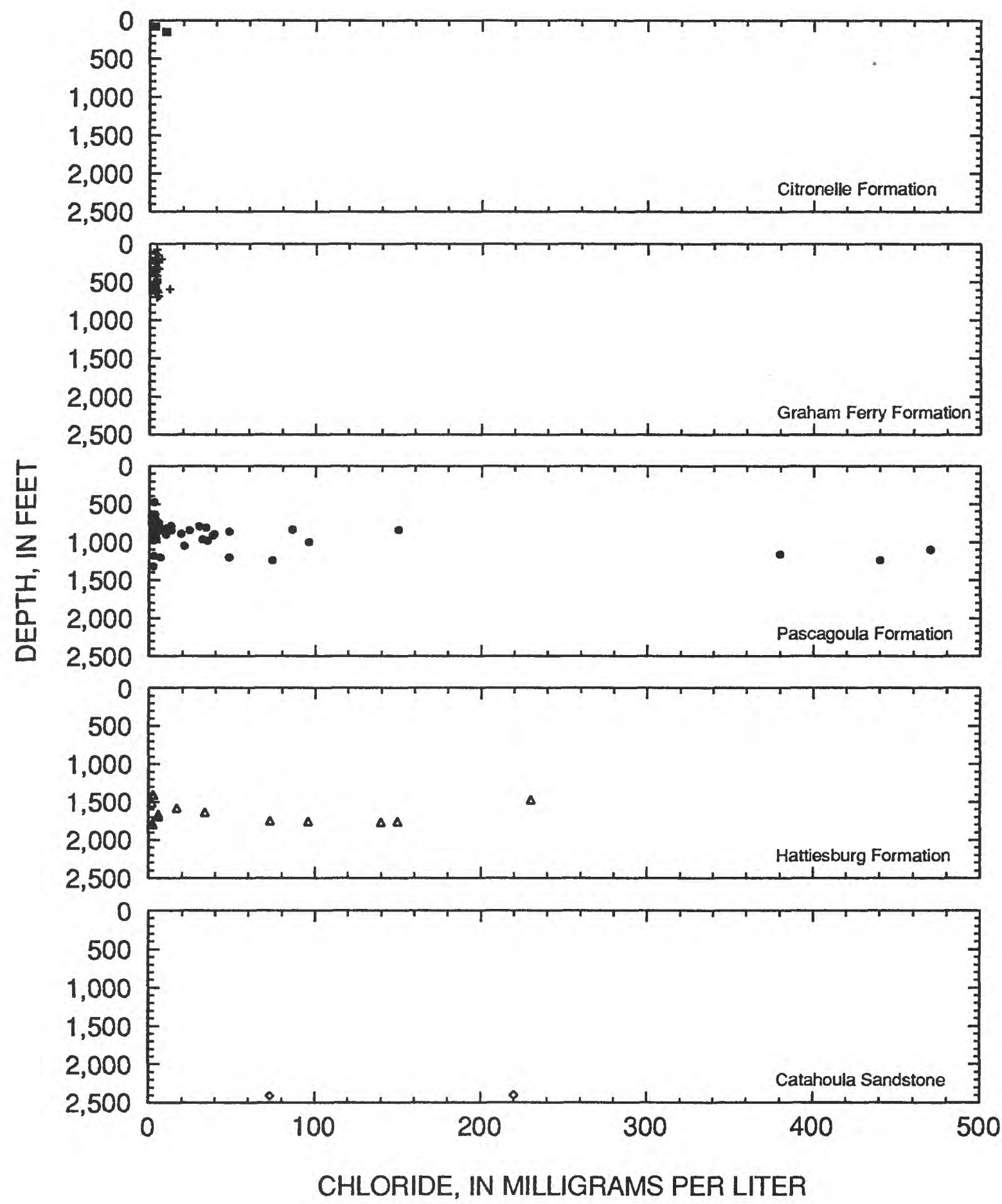

Figure 11.-Relation of depth and chloride concentration in ground water in Harrison County, Mississippi, during 1993. 
Table 1. Summary of site information for wells in Harrison County, Mississippi

[Formations: CRNL, Citronelle; GRMF, Graham Ferry; PCGL, Pascagoula; HBRG, Hattiesburg; CTHL, Catahoula Sandstone; --, no data]

\begin{tabular}{|c|c|c|c|c|c|c|}
\hline $\begin{array}{c}\text { Site } \\
\text { number }\end{array}$ & & $\begin{array}{l}\text { Local number } \\
\text { and owner or } \\
\text { operator }\end{array}$ & $\begin{array}{l}\text { Station } \\
\text { number }\end{array}$ & $\begin{array}{l}\text { Depth } \\
\text { of } \\
\text { well } \\
\text { (feet) }\end{array}$ & $\begin{array}{c}\text { Top } \\
\text { of open } \\
\text { interval } \\
\text { (feet) }\end{array}$ & $\begin{array}{l}\text { Geologic } \\
\text { unit } \\
\text { code }\end{array}$ \\
\hline 1 & A22 & Virgil Shows & 303726089160001 & 310 & -- & GRMF \\
\hline 2 & A42 & John Biskey & 303457089152701 & 120 & 110 & GRMF \\
\hline 3. & B4 & Oscar Ladner & 303439089130901 & 227 & -- & GRMF \\
\hline 4 & B5 & Niron Ladner & 303513089122401 & 330 & -- & GRMF \\
\hline 5 & B9 & William Dickens & 303632089103701 & 257 & -- & GRMF \\
\hline 6 & B10 & F Ladner & 303610089103601 & 200 & -- & GRMF \\
\hline 7 & B12 & Mary House & 303911089090801 & 230 & -- & GRMF \\
\hline 8 & B85 & Louis Simmons & 303809089110001 & 74 & 69 & GRMF \\
\hline 9 & B87 & Richard Ladner & 300610089103601 & 322 & 317 & GRMF \\
\hline 10 & B126 & Clarence Hall & 303919089095301 & 180 & 170 & GRMF \\
\hline 11 & B129 & Bryan Wallace & 303736089095501 & 310 & 295 & GRMF \\
\hline 12 & B161 & Billy Hickman & 303822089120101 & 210 & 200 & GRMF \\
\hline 13 & B166 & Gene Breland & 303827089102902 & 140 & -- & GRMF \\
\hline 14 & C018 & U S Forest Service & 303732089032201 & 638 & 588 & PCGL \\
\hline 15 & C19 & U S Forest Service & 303732089032101 & 230 & -- & GRMF \\
\hline 16 & $\mathrm{C} 27$ & John Alliston & 303527089075001 & 678 & 668 & PCGL \\
\hline 17 & $\mathrm{C} 81$ & Saucier Utility & 303809089081101 & 776 & 746 & PCGL \\
\hline 18 & $\mathrm{C} 120$ & Viola Mason & 303651089054801 & 1,510 & 1,480 & HBRG \\
\hline 19 & C134 & Paul Roberts & 303532089071802 & 300 & 290 & GRMF \\
\hline 20 & C146 & Clifford Vogle & 303540089024801 & 476 & 466 & PCGL \\
\hline 21 & C183 & Buddy Conway & 303730089060001 & 189 & 179 & GRMF \\
\hline 22 & C219 & Church of Christ & 303841089074801 & 237 & 227 & GRMF \\
\hline 23 & C274 & Saucier Utility & 303806089080301 & 1,410 & 1,350 & HBRG \\
\hline 24 & C286 & Larry Haley & 303851089043301 & 730 & 710 & PCGL \\
\hline 25 & C287 & U S Forest Service & 303405089074601 & 875 & 850 & PCGL \\
\hline 26 & C288 & U S Forest Service & 303736089032701 & 645 & 620 & PCGL \\
\hline 27 & D11 & Eldon Mallett & 303858088542001 & 80 & -- & CRNL \\
\hline 28 & D19 & Preston Fayard & 303530088595201 & 326 & 316 & GRMF \\
\hline 29 & D26 & L W Parker & 303335088593001 & 705 & 675 & PCGL \\
\hline 30 & D34 & E E Fayard & 303548088593201 & 215 & 210 & GRMF \\
\hline
\end{tabular}


Table 1. Summary of site information for wells in Harrison County, Mississippi--Continued

\begin{tabular}{|c|c|c|c|c|c|c|}
\hline $\begin{array}{c}\text { Site } \\
\text { number }\end{array}$ & & $\begin{array}{l}\text { Local number } \\
\text { and owner or } \\
\text { operator }\end{array}$ & $\begin{array}{l}\text { Station } \\
\text { number }\end{array}$ & $\begin{array}{c}\text { Depth } \\
\text { of } \\
\text { well } \\
\text { (feet) }\end{array}$ & $\begin{array}{c}\text { Top } \\
\text { of open } \\
\text { interval } \\
\text { (feet) }\end{array}$ & $\begin{array}{l}\text { Geologic } \\
\text { unit } \\
\text { code }\end{array}$ \\
\hline 31 & D36 & Kenneth L Gates & 303832089013201 & 252 & 242 & GRMF \\
\hline 32 & D42 & Sydney Mallett & 303957088580201 & 366 & 356 & GRMF \\
\hline 33 & D43 & Jerry Lepoma & 303348089000801 & 900 & -. & PCGL \\
\hline 34 & E91 & Jim Ussery & 303308089170101 & 1,320 & 1,300 & PCGL \\
\hline 35 & E93 & S S Fazzio & 303318089154301 & 315 & 305 & GRMF \\
\hline 36 & E95 & D Drake & 303130089153401 & 420 & 410 & GRMF \\
\hline 37 & E99 & Kenny Fazzio & 303354089161801 & 420 & 400 & GRMF \\
\hline 38 & E100 & Lonnie Necaise & 302751089185101 & 410 & 400 & GRMF \\
\hline 39 & F081 & Foley Lindsey & 303124089110801 & 325 & 320 & GRMF \\
\hline 40 & F148 & Riverline Utility & 303328089101401 & 358 & 319 & GRMF \\
\hline 41 & F287 & Kevin Morris & 303110089094201 & 635 & 625 & GRMF \\
\hline 42 & F333 & Michael Memorial & 302946089094201 & 520 & 500 & GRMF \\
\hline 43 & F335 & Lizana Fire Dept & 303155089140101 & 560 & 540 & GRMF \\
\hline 44 & F336 & Bill Stephens & 302838089122201 & 980 & 960 & PCGL \\
\hline 45 & F338 & International Paper & 302650089115101 & 202 & 192 & GRMF \\
\hline 46 & G024 & MS Fish Hatchery & 303115089070904 & 785 & 725 & PCGL \\
\hline 47 & G146 & MS Fish Hatchery & 303054089070101 & 420 & 400 & GRMF \\
\hline 48 & G239 & B L S Inc & 302908089064301 & 520 & 460 & GRMF \\
\hline 49 & G425 & Orange Grove & 302825089042001 & 700 & 650 & PCGL \\
\hline 50 & G428 & Johnson & 303048089071701 & 1,800 & -- & HBRG \\
\hline 51 & G429 & Orange Grove & 302934089053501 & 690 & 610 & PCGL \\
\hline 52 & G430 & W Ladner & 303046089052301 & 1,780 & 1,760 & HBRG \\
\hline 53 & $\mathrm{H} 48$ & R H Ladner & 303009088540601 & 840 & 830 & PCGL \\
\hline 54 & $\mathrm{H} 265$ & C M Plummer & 303114089582202 & 794 & 754 & PCGL \\
\hline 55 & $\mathrm{H} 270$ & Doug Young & 303025088542001 & 836 & 816 & PCGL \\
\hline 56 & J192 & Highland Baptist & 302540089143601 & 540 & 525 & GRMF \\
\hline 57 & 211 & G W Byrne & 302447089165201 & 2,410 & 2,350 & CTHL \\
\hline 58 & $\mathrm{~J} 212$ & DuPont & 302306089182801 & 1,760 & 1,660 & HBRG \\
\hline 59 & $\mathrm{~J} 213$ & DuPont & 302308089183101 & 1,770 & 1,640 & HBRG \\
\hline 60 & $\mathrm{~J} 216$ & Waste Management & 302536089162601 & 546 & 506 & GRMF \\
\hline 61 & K4 & MS Housing & 302305089082401 & 785 & 765 & PCGL \\
\hline 62 & K325 & Ricky Lee & 302741089113401 & 1,400 & 1,390 & HBRG \\
\hline 63 & K326 & Deep South Trucking & 302450089112701 & 640 & 620 & PCGL \\
\hline 64 & $\mathrm{~L} 2$ & Gulfport & 302220089055401 & 815 & 755 & PCGL \\
\hline 65 & L14 & Gulfport & 302330089053001 & 763 & 703 & PCGL \\
\hline
\end{tabular}


Table 1. Summary of site information for wells in Harrison County, Mississippi--Continued

\begin{tabular}{|c|c|c|c|c|c|c|}
\hline $\begin{array}{c}\text { Site } \\
\text { number }\end{array}$ & & $\begin{array}{l}\text { Local number } \\
\text { and owner or } \\
\text { operator }\end{array}$ & $\begin{array}{l}\text { Station } \\
\text { number }\end{array}$ & $\begin{array}{c}\text { Depth } \\
\text { of } \\
\text { well } \\
\text { (feet) } \\
\end{array}$ & $\begin{array}{c}\text { Top } \\
\text { of open } \\
\text { interval } \\
\text { (feet) }\end{array}$ & $\begin{array}{l}\text { Geologic } \\
\text { unit } \\
\text { code }\end{array}$ \\
\hline 66 & L15 & Gulfport & 302230089034202 & 752 & 689 & PCGL \\
\hline 67 & L17 & Gulfport & 302206089074505 & 848 & 768 & PCGL \\
\hline 68 & L18 & Orange Grove & 302712089052401 & 765 & 715 & PCGL \\
\hline 69 & L34 & Harrison County & 302602089051301 & 584 & 514 & GRMF \\
\hline 70 & L35 & Dedeaux Utility & 302645089032701 & 730 & 680 & PCGL \\
\hline 71 & L160 & U S Navy & 302252089062001 & 1,200 & 1,130 & PCGL \\
\hline 72 & L161 & U S Navy & 302748089074501 & 850 & -- & PCGL \\
\hline 73 & $\mathrm{~L} 231$ & Orange Grove & 302810089060002 & 697 & 647 & PCGL \\
\hline 74 & L330 & Dedeaux Utility & 302700089030801 & 742 & 678 & PCGL \\
\hline 75 & L405 & Gulfport & 302357089064201 & 795 & 715 & PCGL \\
\hline 76 & L411 & Harrison County & 302603089050201 & 711 & 661 & PCGL \\
\hline 77 & L426 & Gulfport & 302252089053301 & 926 & 886 & PCGL \\
\hline 78 & L430 & U S Navy & 302251089062901 & 746 & 686 & PCGL \\
\hline 79 & L433 & U S Navy & 302249089073701 & 720 & 649 & PCGL \\
\hline 80 & L454 & Carl McGill & 302403089141801 & 2,400 & 2,380 & CTHL \\
\hline 81 & L654 & Dolan Trailer Park & 302410089082201 & 1,690 & 1,670 & HBRG \\
\hline 82 & L660 & Dedeaux Utility & 302659089030801 & 741 & 690 & PCGL \\
\hline 83 & L663 & Sterling Drug & 302331089053401 & 1,180 & 1,120 & PCGL \\
\hline 84 & L667 & Gulfport & 302152089064901 & 952 & 772 & PCGL \\
\hline 85 & L669 & Fore Concrete & 302656089054901 & 1,660 & 1,600 & HBRG \\
\hline 86 & L670 & Orange Grove & 302646089053001 & 750 & 670 & PCGL \\
\hline 87 & M4 & Biloxi & 302342088563003 & 1,200 & 1,120 & PCGL \\
\hline 88 & M6 & Biloxi & 302410088533607 & 463 & 384 & GRMF \\
\hline 89 & M23 & MS Power & 302611089014801 & 755 & 695 & PCGL \\
\hline 90 & M24 & MS Power & 302615089011503 & 845 & 785 & PCGL \\
\hline 91 & M26 & Harrison County & 302440089014501 & 752 & 679 & PCGL \\
\hline 92 & M31 & Oakleigh Manor & 302702089011401 & 510 & 486 & GRMF \\
\hline 93 & M33 & Biloxi & 302455088584001 & 700 & 660 & GRMF \\
\hline 94 & M40 & Biloxi & 302454088580001 & 654 & 604 & GRMF \\
\hline 95 & M75 & U S Air Force & 302451088550501 & 610 & 570 & GRMF \\
\hline 96 & M76 & US Air Force & 302432088541601 & 630 & 590 & GRMF \\
\hline 97 & M79 & U S Air Force & 302354088555101 & 640 & 600 & GRMF \\
\hline 98 & M196 & Biloxi & 302624089000001 & 850 & 840 & PCGL \\
\hline 99 & M266 & James Talley & 302557088530301 & 1,236 & 1,210 & PCGL \\
\hline 100 & M300 & Oakleigh Utility & 302712089012001 & 508 & 488 & GRMF \\
\hline
\end{tabular}


Table 1. Summary of site information for wells in Harrison County, Mississippi--Continued

\begin{tabular}{|c|c|c|c|c|c|c|}
\hline $\begin{array}{c}\text { Site } \\
\text { number }\end{array}$ & & $\begin{array}{l}\text { Local number } \\
\text { and owner or } \\
\text { operator }\end{array}$ & $\begin{array}{l}\text { Station } \\
\text { number }\end{array}$ & $\begin{array}{c}\text { Depth } \\
\text { of } \\
\text { well } \\
\text { (feet) }\end{array}$ & $\begin{array}{c}\text { Top } \\
\text { of open } \\
\text { interval } \\
\text { (feet) } \\
\end{array}$ & $\begin{array}{l}\text { Geologic } \\
\text { unit } \\
\text { code }\end{array}$ \\
\hline 101 & M351 & Biloxi & 302350088570501 & 846 & 796 & PCGL \\
\hline 102 & M380 & Gulfport & 302457089014501 & 786 & 736 & PCGL \\
\hline 103 & M397 & Gulfport & 302346089001501 & 830 & 770 & PCGL \\
\hline 104 & M428 & Imperial Estate & 302632088565001 & 593 & 563 & GRMF \\
\hline 105 & M519 & Biloxi & 302554088580401 & 546 & 486 & GRMF \\
\hline 106 & M550 & Biloxi & 302456088541304 & 634 & 564 & GRMF \\
\hline 107 & M576 & Biloxi & 302404088590301 & 848 & 788 & PCGL \\
\hline 108 & M643 & D’Iberville & 302410088531501 & 840 & 747 & PCGL \\
\hline 109 & M644 & Gulfport & 302352089015701 & 820 & 740 & PCGL \\
\hline 110 & M648 & Gulfport & 302409089002101 & 845 & 745 & PCGL \\
\hline 111 & M657 & Gulfport & 302304089020301 & 900 & 820 & PCGL \\
\hline 112 & M661 & Biloxi & 302555088555401 & 550 & 500 & GRMF \\
\hline 113 & M674 & Biloxi & 302552088580401 & 593 & 533 & GRMF \\
\hline 114 & M696 & Biloxi & 302428088531501 & 915 & 855 & PCGL \\
\hline 115 & M698 & Biloxi & 302356088595701 & 819 & 758 & PCGL \\
\hline 116 & M703 & Biloxi & 302610088580201 & 845 & 755 & PCGL \\
\hline 117 & M704 & Biloxi & 302540088565001 & 593 & 533 & GRMF \\
\hline 118 & M709 & MS Coast Coliseum & 302340088582601 & 150 & 120 & CRNL \\
\hline 119 & M710 & Gulf Orleans & 302810088545201 & 332 & 282 & GRMF \\
\hline 120 & M711 & John Sherman & 302812088592101 & 1,472 & 1,457 & HBRG \\
\hline 121 & M714 & Galion Utility & 302645088540301 & 810 & 750 & PCGL \\
\hline 122 & M722 & Dels Seaway & 302439088531301 & 1,240 & -- & PCGL \\
\hline 123 & M727 & Biloxi & 302430088574901 & 649 & 588 & GRMF \\
\hline 124 & M729 & Biloxi & 302359088522001 & 895 & 825 & PCGL \\
\hline 125 & M730 & Biloxi & 302348088560501 & 845 & 785 & PCGL \\
\hline 126 & M734 & Dels Seaway & 302438088531001 & 1,160 & 1,110 & PCGL \\
\hline 127 & M739 & Citanovitch Packing & 302443088525001 & 1,100 & 1,050 & PCGL \\
\hline 128 & M741 & Rolling Hills & 302627088545201 & 1,000 & -- & PCGL \\
\hline 129 & M744 & Cedar Lake Trailer Park & 302623088554801 & 846 & 806 & PCGL \\
\hline 130 & N102 & Pass Christian & 301838089163201 & 966 & 906 & PCGL \\
\hline 131 & N104 & DuPont & 302258089183101 & 1,750 & 1,600 & HBRG \\
\hline 132 & N166 & DuPont & 302233089191501 & 684 & 644 & GRMF \\
\hline 133 & N291 & DuPont & 302300089180601 & 1,760 & 1,620 & HBRG \\
\hline 134 & N313 & Pass Christian & 301832089171301 & 982 & 912 & PCGL \\
\hline 135 & N327 & Pass Christian & 301917089153101 & 858 & 823 & PCGL \\
\hline
\end{tabular}


Table 1. Summary of site information for wells in Harrison County, Mississippi--Continued

\begin{tabular}{|c|c|c|c|c|c|c|}
\hline $\begin{array}{c}\text { Site } \\
\text { number }\end{array}$ & & $\begin{array}{l}\text { Local number } \\
\text { and owner or } \\
\text { operator }\end{array}$ & $\begin{array}{l}\text { Station } \\
\text { number }\end{array}$ & $\begin{array}{c}\text { Depth } \\
\text { of } \\
\text { well } \\
\text { (feet) }\end{array}$ & $\begin{array}{c}\text { Top } \\
\text { of open } \\
\text { interval } \\
\text { (feet) }\end{array}$ & $\begin{array}{l}\text { Geologic } \\
\text { unit } \\
\text { code }\end{array}$ \\
\hline 136 & O6 & Pass Christian & 301936089142403 & 891 & 841 & PCGL \\
\hline 137 & O8 & Long Beach & 302200089100601 & 611 & 571 & GRMF \\
\hline 138 & 011 & Long Beach & 302215089102001 & 590 & 550 & GRMF \\
\hline 139 & O123 & Long Beach & 302020089110001 & 873 & 803 & PCGL \\
\hline 140 & O125 & Margot Gack & 302134089141501 & 1,580 & 1,550 & HBRG \\
\hline 141 & $\mathrm{O} 251$ & Long Beach & 302207089100501 & 850 & 790 & PCGL \\
\hline 142 & $\mathrm{O} 268$ & Long Beach & 302059089090601 & 876 & 816 & PCGL \\
\hline 143 & $\mathrm{O} 296$ & Long Beach & 302139089084401 & 862 & 778 & PCGL \\
\hline 144 & $\mathrm{O} 307$ & Long Beach & 302204089102701 & 1,640 & 1,560 & HBRG \\
\hline 145 & $\mathrm{O} 309$ & Coast Water Works & 302023089135101 & 1,050 & 1,010 & PCGL \\
\hline
\end{tabular}


Table 2. Water-quality data collected from wells in Harrison County, Mississippi, during 1993

[Formations: CRNL, Citronelle; GRMF, Graham Ferry; PCGL, Pascagoula; HBRG, Hattiesburg; CTHL, Catahoula Sandstone; $\mu \mathrm{S} / \mathrm{cm}$, microsiemens per centimeter at 25 degrees Celsius; ROE, residue on evaporation at 180 degrees Celsius; $\mathrm{mg} / \mathrm{L}$, milligrams per liter; $\mathrm{N}$, nitrogen; $<$, less than; --, no data]

\begin{tabular}{|c|c|c|c|c|c|c|c|c|c|c|c|}
\hline $\begin{array}{l}\text { Site } \\
\text { number }\end{array}$ & $\begin{array}{c}\text { Local } \\
\text { number }\end{array}$ & $\begin{array}{l}\text { Geologic } \\
\text { unit } \\
\text { code }\end{array}$ & Date & $\begin{array}{r}\mathrm{Sp} \\
\text { cond } \\
(\mu \mathrm{S} \\
\text { field } \\
\end{array}$ & & field & lab & $\begin{array}{c}\text { Color } \\
\text { (platinum- } \\
\text { cobalt } \\
\text { units) } \\
\end{array}$ & $\begin{array}{c}\mathrm{ROE} \\
(\mathrm{mg} / \mathrm{L})\end{array}$ & $\begin{array}{l}\text { Chloride } \\
\text { (mg/L ) }\end{array}$ & $\begin{array}{c}\text { Nitrite } \\
\text { plus } \\
\text { itrate, as } N \\
(\mathrm{mg} / \mathrm{L}) \\
\end{array}$ \\
\hline 1 & A22 & GRMF & July 19 & -- & 127 & 6.8 & 6.9 & $<5$ & 125 & 3.7 & $<0.02$ \\
\hline 2 & A42 & GRMF & July 19 & 78 & 75 & 6.7 & 7.3 & $<5$ & 111 & 3.4 & $<.02$ \\
\hline 3 & B4 & GRMF & July 7 & 23 & 27 & 5.6 & 6.2 & $<5$ & 30 & 3.6 & .02 \\
\hline 4 & B5 & GRMF & July 7 & 13 & 15 & 5.5 & 5.9 & $<5$ & 20 & 2.7 & .02 \\
\hline 5 & B9 & GRMF & July 7 & 119 & 124 & 6.8 & 7.3 & 50 & 129 & 3.5 & $<.02$ \\
\hline 6 & B10 & GRMF & July 7 & 154 & 155 & 7.2 & 7.6 & $<5$ & 151 & 3.6 & $<.02$ \\
\hline 7 & B12 & GRMF & July 7 & 118 & 107 & 6.6 & 7.0 & 120 & 121 & 2.9 & $<.02$ \\
\hline 8 & B85 & GRMF & July 2 & 54 & 53 & 5.2 & 6.6 & $<5$ & 91 & 4.1 & .05 \\
\hline 9 & B87 & GRMF & July 7 & 108 & 122 & 6.8 & 7.1 & $<5$ & 130 & 3.5 & $<.02$ \\
\hline 10 & B126 & GRMF & July 7 & 121 & 120 & 6.7 & 7.2 & $<5$ & 128 & 3.1 & $<.02$ \\
\hline 11 & B129 & GRMF & July 2 & 185 & 192 & 7.1 & 7.9 & $<5$ & 163 & 4.0 & $<.02$ \\
\hline 12 & B161 & GRMF & July 7 & 158 & 157 & 6.8 & 7.4 & $<5$ & 142 & 3.8 & $<.02$ \\
\hline 13 & B166 & GRMF & July 2 & 30 & 34 & 5.5 & 5.9 & $<5$ & 48 & 4.8 & .59 \\
\hline 14 & C18 & PCGL & June 30 & 268 & 270 & 8.3 & 8.1 & $<5$ & 193 & 2.4 & $<.02$ \\
\hline 15 & C19 & GRMF & June 30 & 179 & 170 & 6.9 & 7.2 & $<5$ & 160 & 2.8 & .04 \\
\hline 16 & $\mathrm{C} 27$ & PCGL & June 30 & 243 & 262 & 9.0 & 8.8 & $<5$ & 172 & 2.3 & $<.02$ \\
\hline 17 & C81 & PCGL & June 30 & 238 & 227 & 8.7 & 8.6 & $<5$ & 176 & 2.7 & $<.02$ \\
\hline 18 & C120 & HBRG & June 30 & 230 & 225 & 8.6 & 8.2 & $<5$ & 150 & 1.9 & $<.02$ \\
\hline 19 & C134 & GRMF & July 1 & 113 & 108 & 6.5 & 6.9 & $<5$ & 120 & 3.1 & $<.02$ \\
\hline 20 & C146 & PCGL & July 1 & 111 & 112 & 6.5 & 7.1 & 60 & 129 & 2.9 & $<.02$ \\
\hline 21 & C183 & GRMF & July 2 & 167 & 170 & 6.7 & 7.6 & $<5$ & 145 & 3.3 & $<.02$ \\
\hline 22 & C219 & GRMF & June 30 & 99 & 102 & 6.7 & 7.0 & $<5$ & 120 & 3.0 & $<.02$ \\
\hline 23 & $\mathrm{C} 274$ & HBRG & June 30 & 253 & 250 & 8.6 & 8.6 & $<5$ & 180 & 3.0 & $<.02$ \\
\hline 24 & C286 & PCGL & July 1 & 303 & 309 & 8.9 & 8.7 & $<5$ & 196 & 2.4 & $<.02$ \\
\hline 25 & C287 & PCGL & July 1 & 280 & 283 & 9.0 & 8.9 & $<5$ & 186 & 2.4 & $<.02$ \\
\hline 26 & C288 & PCGL & June 30 & 292 & 288 & 8.5 & 8.3 & $<5$ & 199 & 2.3 & $<.02$ \\
\hline 27 & D11 & CRNL & July 1 & 23 & 21 & 5.5 & 6.1 & $<5$ & 37 & 3.1 & $<.02$ \\
\hline 28 & D19 & GRMF & July 1 & 141 & 130 & 7.0 & 7.1 & 30 & 149 & 3.0 & $<.02$ \\
\hline 29 & D26 & PCGL & July 1 & 173 & 176 & 6.9 & 7.5 & $<5$ & 172 & 2.7 & $<.02$ \\
\hline 30 & D34 & GRMF & July 1 & 177 & 173 & 7.2 & 7.6 & $<5$ & 155 & 3.2 & $<.02$ \\
\hline
\end{tabular}


Table 2. Water-quality data collected from wells in Harrison County, Mississippi, during 1993--Continued

\begin{tabular}{|c|c|c|c|c|c|c|c|c|c|c|c|}
\hline $\begin{array}{l}\text { Site } \\
\text { number }\end{array}$ & $\begin{array}{l}\text { Local } \\
\text { number }\end{array}$ & $\begin{array}{l}\text { Geologic } \\
\text { unit } \\
\text { code }\end{array}$ & Date & $\begin{array}{r}\mathrm{Sp} \\
\text { cond } \\
(\mu \mathrm{S} \\
\text { field } \\
\end{array}$ & $\begin{array}{l}\text { fic } \\
\text { ance } \\
\text { m) } \\
\text { lab } \\
\end{array}$ & field & lab & $\begin{array}{c}\text { Color } \\
\text { (platinum- } \\
\text { cobalt } \\
\text { units) } \\
\end{array}$ & $\begin{array}{c}\mathrm{ROE} \\
(\mathrm{mg} / \mathrm{L})\end{array}$ & $\begin{array}{r}\text { Chloride } \\
\text { (mg/L) } \\
\text { n }\end{array}$ & $\begin{array}{c}\text { Nitrite } \\
\text { plus } \\
\text { hitrate, as } \mathrm{N} \\
(\mathrm{mg} / \mathrm{L}) \\
\end{array}$ \\
\hline 31 & D36 & GRMF & July 1 & 114 & 111 & 6.6 & 6.8 & 10 & 128 & 3.4 & $<0.02$ \\
\hline 32 & D42 & GRMF & July 1 & 339 & 338 & 7.5 & 7.7 & $<5$ & 233 & 2.5 & $<.02$ \\
\hline 33 & D43 & PCGL & July 1 & 600 & 595 & 8.9 & 8.6 & 25 & 382 & 3.1 & $<.02$ \\
\hline 34 & E91 & PCGL & July 19 & 322 & 345 & 9.3 & 9.0 & 5 & 215 & 2.3 & $<.02$ \\
\hline 35 & E93 & GRMF & July 19 & 163 & 198 & 7.5 & 7.6 & $<5$ & 157 & 4.0 & .08 \\
\hline 36 & E95 & GRMF & July 19 & 227 & 223 & 8.4 & 8.3 & $<5$ & 142 & 4.0 & $<.02$ \\
\hline 37 & E99 & GRMF & July 19 & 170 & 156 & 6.9 & 7.6 & 50 & 143 & 3.4 & $<.02$ \\
\hline 38 & E100 & GRMF & July 21 & 190 & 189 & 7.3 & 7.5 & 20 & 154 & 3.4 & $<.02$ \\
\hline 39 & F81 & GRMF & July 20 & 244 & 246 & 8.1 & 8.1 & $<5$ & 164 & 5.4 & $<.02$ \\
\hline 40 & F148 & GRMF & July 21 & 166 & 163 & 7.4 & 7.8 & $<5$ & 141 & 3.7 & $<.02$ \\
\hline 41 & F287 & GRMF & July 20 & 147 & 145 & 6.7 & 7.0 & 5 & 140 & 3.5 & $<.02$ \\
\hline 42 & F333 & GRMF & July 20 & 198 & 194 & 7.6 & 7.8 & $<5$ & 149 & 3.7 & $<.02$ \\
\hline 43 & F335 & GRMF & July 20 & 196 & 193 & 7.5 & 7.6 & $<5$ & 159 & 3.4 & $<.02$ \\
\hline 44 & F336 & PCGL & July 20 & 384 & 387 & 9.3 & 8.9 & 10 & 237 & 2.4 & .06 \\
\hline 45 & F338 & GRMF & July 20 & 118 & 113 & 6.5 & 7.0 & 10 & 141 & 6.8 & $<.02$ \\
\hline 46 & G24 & PCGL & July 8 & 186 & 184 & 8.6 & 8.4 & $<5$ & 165 & 3.4 & .03 \\
\hline 47 & G146 & GRMF & July 8 & 169 & 169 & 7.3 & 7.3 & $<5$ & 140 & 3.6 & $<.02$ \\
\hline 48 & G239 & GRMF & July 6 & 180 & 180 & 7.5 & 7.6 & $<5$ & 152 & 3.7 & $<.02$ \\
\hline 49 & G425 & PCGL & July 6 & 291 & 295 & 8.9 & 8.7 & $<5$ & 200 & 2.7 & $<.02$ \\
\hline 50 & G428 & HBRG & July 19 & -- & 280 & 9.0 & 8.5 & 5 & 182 & 2.4 & $<.02$ \\
\hline 51 & G429 & PCGL & July 6 & 268 & 265 & 9.0 & 8.6 & $<5$ & 182 & 3.0 & .03 \\
\hline 52 & G430 & HBRG & June 29 & 292 & 298 & 8.8 & 8.7 & 5 & 194 & 2.3 & $<.02$ \\
\hline 53 & $\mathrm{H} 48$ & PCGL & July 20 & 918 & 919 & 8.8 & 8.6 & 10 & 539 & 150 & $<.02$ \\
\hline 54 & $\mathrm{H} 265$ & PCGL & July 20 & 444 & 434 & 9.1 & 8.5 & $<5$ & 278 & 30 & .03 \\
\hline 55 & $\mathrm{H} 270$ & PCGL & July 20 & 657 & 667 & 8.9 & 8.7 & 10 & 421 & 86 & $<.02$ \\
\hline 56 & J192 & GRMF & July 20 & 207 & 206 & 7.1 & 7.5 & $<5$ & 164 & 3.3 & $<.02$ \\
\hline 57 & $\mathrm{~J} 211$ & CTHL & July 21 & 590 & 592 & 8.9 & 8.5 & 10 & 339 & 73 & $<.02$ \\
\hline 58 & $\mathrm{~J} 212$ & HBRG & July 7 & 844 & 823 & 8.7 & 8.1 & $<5$ & 456 & 150 & $<.02$ \\
\hline 59 & $\mathrm{~J} 213$ & HBRG & July 7 & 770 & 763 & 9.1 & 8.5 & $<5$ & 422 & 140 & $<.02$ \\
\hline 60 & $\mathrm{~J} 216$ & GRMF & July 9 & 202 & 202 & 7.4 & 7.5 & $<5$ & 165 & 3.3 & $<.02$ \\
\hline 61 & $\mathrm{~K} 4$ & PCGL & July 20 & 234 & 233 & 8.2 & 8.0 & $<5$ & 168 & 3.9 & $<.02$ \\
\hline 62 & K325 & HBRG & July 19 & 369 & 369 & 9.2 & 9.0 & 5 & 254 & 2.3 & $<.02$ \\
\hline 63 & K326 & PCGL & July 7 & 207 & 206 & 8.1 & 7.8 & $<5$ & 170 & 3.4 & $<.02$ \\
\hline 64 & L2 & PCGL & July 7 & 307 & 297 & 8.2 & 8.8 & $<5$ & 202 & 4.3 & $<.02$ \\
\hline 65 & L14 & PCGL & July 7 & 247 & 244 & 8.0 & 8.8 & $<5$ & 194 & 3.3 & $<.02$ \\
\hline
\end{tabular}


Table 2. Water-quality data collected from wells in Harrison County, Mississippi, during 1993--Continued

\begin{tabular}{|c|c|c|c|c|c|c|c|c|c|c|c|}
\hline $\begin{array}{l}\text { Site } \\
\text { number }\end{array}$ & $\begin{array}{l}\text { Local } \\
\text { number }\end{array}$ & $\begin{array}{l}\text { Geologic } \\
\text { unit } \\
\text { code }\end{array}$ & Date & $\begin{array}{r}\mathrm{Sp} \\
\text { condi } \\
(\mu \mathrm{S} \\
\text { field } \\
\end{array}$ & $\begin{array}{l}\text { ific } \\
\text { ctance } \\
\mathrm{cm}) \\
\text { lab } \\
\end{array}$ & field $^{p}$ & lab & $\begin{array}{l}\text { Color } \\
\text { (platinum- } \\
\text { cobalt } \\
\text { units) } \\
\end{array}$ & $\begin{array}{c}\mathrm{ROE} \\
(\mathrm{mg} / \mathrm{L})\end{array}$ & $\begin{array}{l}\text { Chloride } \\
(\mathrm{mg} / \mathrm{L})\end{array}$ & $\begin{array}{l}\text { Nitrite } \\
\text { plus } \\
\text { itrate, as } \mathrm{N} \\
(\mathrm{mg} / \mathrm{L}) \\
\end{array}$ \\
\hline 66 & L15 & PCGL & July 7 & 268 & 273 & 8.6 & 8.5 & $<5$ & $190^{\circ}$ & 3.8 & $<0.02$ \\
\hline 67 & L17 & PCGL & July 7 & 252 & 253 & 8.0 & 8.0 & $<5$ & 184 & 3.9 & $<.02$ \\
\hline 68 & L18 & PCGL & July 6 & 240 & 236 & 8.7 & 8.4 & $<5$ & 174 & 3.6 & $<.02$ \\
\hline 69 & L34 & GRMF & July 20 & 434 & 429 & 9.1 & 8.8 & 5 & 296 & 2.6 & $<.02$ \\
\hline 70 & L35 & PCGL & July 21 & 316 & 315 & 9.1 & 8.9 & $<5$ & 217 & 2.6 & $<.02$ \\
\hline 71 & L160 & PCGL & July 7 & 246 & 247 & 7.7 & 8.0 & $<5$ & 189 & 6.6 & $<.02$ \\
\hline 72 & L161 & PCGL & July 7 & 290 & 290 & 8.9 & 8.7 & $<5$ & 206 & 4.0 & $<.02$ \\
\hline 73 & L231 & PCGL & July 6 & 280 & 228 & 8.6 & 8.2 & $<5$ & 172 & 3.7 & $<.02$ \\
\hline 74 & L330 & PCGL & July 21 & 381 & 384 & 9.2 & 8.9 & $<5$ & 255 & 2.6 & $<.02$ \\
\hline 75 & L405 & PCGL & July 7 & 294 & 299 & 8.8 & 8.6 & $<5$ & 206 & 2.9 & $<.02$ \\
\hline 76 & L411 & PCGL & July 20 & 254 & 251 & 8.9 & 8.4 & $<5$ & 213 & 3.6 & $<.02$ \\
\hline 77 & L426 & PCGL & July 7 & 343 & 346 & 9.1 & 8.9 & $<5$ & 224 & 3.3 & $<.02$ \\
\hline 78 & L430 & PCGL & July 7 & 217 & 243 & 8.3 & 8.4 & $<5$ & 183 & 4.6 & $<.02$ \\
\hline 79 & L433 & PCGL & July 7 & 241 & 237 & 7.4 & 7.8 & $<5$ & 188 & 4.2 & $<.02$ \\
\hline 80 & L454 & CTHL & July 6 & 1,130 & 1,110 & 9.0 & 8.7 & 10 & 638 & 220 & $<.02$ \\
\hline 81 & L654 & HBRG & June 30 & 357 & 355 & 8.8 & 8.8 & $<5$ & 231 & 5.9 & $<.02$ \\
\hline 82 & L660 & PCGL & July 21 & 345 & 342 & 9.0 & 8.8 & $<5$ & 234 & 2.8 & $<.02$ \\
\hline 83 & L663 & PCGL & July 19 & 452 & 454 & 9.1 & 8.9 & 15 & 280 & 2.6 & $<.02$ \\
\hline 84 & L667 & PCGL & July 7 & 277 & 281 & 8.7 & 8.5 & $<5$ & 200 & 4.0 & $<.02$ \\
\hline 85 & L669 & HBRG & July 19 & -- & 248 & 8.1 & 8.1 & $<5$ & 196 & 5.8 & .03 \\
\hline 86 & L670 & PCGL & July 6 & 253 & 239 & 8.6 & 8.2 & $<5$ & 186 & 3.5 & $<.02$ \\
\hline 87 & M4 & PCGL & July 8 & 560 & 554 & 8.9 & 8.7 & 10 & 331 & 48 & $<.02$ \\
\hline 88 & M6 & GRMF & July 8 & 311 & 314 & 8.7 & 8.5 & $<5$ & 208 & 4.0 & $<.02$ \\
\hline 89 & M23 & PCGL & July 8 & 359 & 354 & 8.9 & 8.8 & $<5$ & 234 & 2.8 & $<.02$ \\
\hline 90 & M24 & PCGL & July 8 & 491 & 491 & 8.9 & 8.8 & 10 & 305 & 13 & -- \\
\hline 91 & M26 & PCGL & July 20 & 207 & 207 & 8.5 & 8.0 & $<5$ & 189 & 5.9 & $<.02$ \\
\hline 92 & M31 & GRMF & July 8 & 317 & 316 & 8.5 & 8.2 & $<5$ & 228 & 3.1 & $<.02$ \\
\hline 93 & M33 & GRMF & July 8 & 327 & 324 & 8.6 & 8.5 & $<5$ & 221 & 4.2 & $<.02$ \\
\hline 94 & M40 & GRMF & July 9 & 279 & 271 & 7.6 & 7.8 & $<5$ & 208 & 3.5 & $<.02$ \\
\hline 95 & M75 & GRMF & July 8 & 263 & 260 & 7.9 & 7.7 & $<5$ & 199 & 4.5 & .85 \\
\hline 96 & M76 & GRMF & July 8 & 226 & 237 & 7.9 & 8.0 & $<5$ & 186 & 4.1 & $<.02$ \\
\hline 97 & M79 & GRMF & July 8 & 318 & 317 & 8.7 & 8.6 & $<5$ & 216 & 5.0 & $<.02$ \\
\hline 98 & M196 & PCGL & July 8 & 316 & 310 & -- & 8.8 & 5 & 197 & 8.8 & .14 \\
\hline 99 & M266 & PCGL & July 20 & 1,830 & 1,880 & 8.9 & 8.7 & 10 & 1,020 & 440 & $<.02$ \\
\hline 100 & M300 & GRMF & July 8 & 347 & 345 & 9.2 & 8.9 & $<5$ & 234 & 2.5 & $<.02$ \\
\hline
\end{tabular}


Table 2. Water-quality data collected from wells in Harrison County, Mississippi, during 1993--Continued

\begin{tabular}{|c|c|c|c|c|c|c|c|c|c|c|c|}
\hline $\begin{array}{l}\text { Site } \\
\text { number }\end{array}$ & $\begin{array}{l}\text { Local } \\
\text { number }\end{array}$ & $\begin{array}{l}\text { Geologic } \\
\text { unit } \\
\text { code }\end{array}$ & Date & $\begin{array}{r}\text { Spe } \\
\text { condu } \\
(\mu S / \\
\text { field } \\
\end{array}$ & $\begin{array}{l}\text { ific } \\
\text { ctance } \\
\mathrm{cm}) \\
\text { lab } \\
\end{array}$ & field & lab & $\begin{array}{c}\text { Color } \\
\text { (platinum- } \\
\text { cobalt } \\
\text { units) } \\
\end{array}$ & $\begin{array}{c}\mathrm{ROE} \\
(\mathrm{mg} / \mathrm{L})\end{array}$ & $\begin{array}{r}\text { Chloride } \\
(\mathrm{mg} / \mathrm{L}) \\
\mathrm{n} \\
\end{array}$ & $\begin{array}{c}\text { Nitrite } \\
\text { plus } \\
\text { citrate, as } \mathrm{N} \\
\text { (mg/L) } \\
\end{array}$ \\
\hline 101 & M351 & PCGL & July 8 & 405 & 399 & 9.0 & 8.8 & 10 & 250 & 7.4 & $<0.02$ \\
\hline 102 & M380 & PCGL & July 8 & 440 & 451 & 8.8 & 8.4 & 20 & 289 & 13 & $<.02$ \\
\hline 103 & M397 & PCGL & July 8 & 369 & 373 & 9.2 & 8.8 & 5 & 243 & 4.2 & $<.02$ \\
\hline 104 & M428 & GRMF & July 21 & 271 & 266 & 7.7 & 7.7 & $<5$ & 202 & 2.9 & $<.02$ \\
\hline 105 & M519 & GRMF & July 8 & -- & 236 & 8.5 & 8.2 & $<5$ & 171 & 2.9 & $<.02$ \\
\hline 106 & M550 & GRMF & July 8 & 280 & 277 & 8.0 & 8.1 & $<5$ & 210 & 4.2 & $<.02$ \\
\hline 107 & M576 & PCGL & July 8 & 389 & 391 & 9.0 & 8.9 & 10 & 249 & 6.8 & $<.02$ \\
\hline 108 & M643 & PCGL & July 20 & 569 & 562 & 9.0 & 8.7 & 10 & 371 & 6.6 & $<.02$ \\
\hline 109 & M644 & PCGL & July 8 & 431 & 438 & 9.2 & 8.7 & 5 & 281 & 10 & $<.02$ \\
\hline 110 & M648 & PCGL & July 8 & 430 & 433 & 9.2 & 8.8 & 10 & 284 & 10 & $<.02$ \\
\hline 111 & M657 & PCGL & July 8 & 425 & 430 & 9.1 & 8.7 & 5 & 281 & 9.8 & $<.02$ \\
\hline 112 & M661 & GRMF & July 9 & 242 & 190 & 7.5 & 7.6 & $<5$ & 168 & 3.3 & $<.02$ \\
\hline 113 & M674 & GRMF & July 8 & 330 & 312 & 9.0 & 8.7 & 5 & 204 & 12 & $<.02$ \\
\hline 114 & M696 & PCGL & July 8 & 532 & 534 & 8.9 & 8.7 & 10 & 325 & 38 & $<.02$ \\
\hline 115 & M698 & PCGL & July 8 & 398 & 396 & 8.9 & 8.8 & $<5$ & 261 & 6.6 & $<.02$ \\
\hline 116 & M703 & PCGL & July 8 & 340 & 328 & 9.0 & 8.7 & $<5$ & 209 & 12 & $<.02$ \\
\hline 117 & M704 & GRMF & July 9 & 370 & 327 & 8.3 & 8.1 & $<5$ & 229 & 3.5 & $<.02$ \\
\hline 118 & M709 & CRNL & July 8 & 360 & 354 & 8.5 & 8.5 & $<5$ & 236 & 9.8 & -- \\
\hline 119 & M710 & GRMF & July 21 & 275 & 273 & 8.5 & 8.2 & $<5$ & 220 & 3.8 & $<.02$ \\
\hline 120 & M711 & HBRG & July 20 & 1,240 & 1,260 & 8.7 & 8.5 & 20 & 711 & 230 & $<.02$ \\
\hline 121 & M714 & PCGL & July 20 & 543 & 539 & 9.1 & 8.8 & 10 & 325 & 34 & $<.02$ \\
\hline 122 & M722 & PCGL & July 8 & 636 & 643 & 9.0 & 8.4 & 10 & 387 & 74 & $<.02$ \\
\hline 123 & M727 & GRMF & July 9 & 230 & 223 & 8.2 & 8.1 & $<5$ & 182 & 3.1 & $<.02$ \\
\hline 124 & M729 & PCGL & July 8 & 538 & 533 & 9.0 & 8.7 & 10 & 336 & 39 & $<.02$ \\
\hline 125 & M730 & PCGL & July 8 & 400 & 394 & 9.0 & 8.8 & $<5$ & 251 & 8.1 & $<.02$ \\
\hline 126 & M734 & PCGL & July 8 & 1,640 & 1,690 & 8.7 & 8.5 & 20 & 921 & 380 & $<.02$ \\
\hline 127 & M739 & PCGL & July 21 & 2,000 & 2,020 & 8.6 & 8.4 & 50 & 1,120 & 470 & $<.02$ \\
\hline 128 & M741 & PCGL & July 21 & 717 & 759 & 9.0 & 8.6 & 10 & 434 & 96 & $<.02$ \\
\hline 129 & M744 & PCGL & July 21 & 430 & 432 & 9.0 & 8.7 & 5 & 272 & 24 & .05 \\
\hline 130 & N102 & PCGL & June 30 & 621 & 622 & 8.7 & 8.6 & 20 & 384 & 32 & $<.02$ \\
\hline 131 & N104 & HBRG & July 7 & 569 & 564 & 8.8 & 8.2 & $<5$ & 319 & 73 & $<.02$ \\
\hline 132 & N166 & GRMF & July 7 & 246 & 241 & 7.8 & 7.8 & 10 & 191 & 5.2 & $<.02$ \\
\hline 133 & N291 & HBRG & July 7 & 638 & 622 & 8.7 & 8.2 & $<5$ & 357 & 96 & $<.02$ \\
\hline 134 & N313 & PCGL & June 30 & 557 & 560 & 8.7 & 8.6 & 15 & 343 & 35 & $<.02$ \\
\hline 135 & N327 & PCGL & June 30 & 636 & 637 & 8.7 & 8.7 & 20 & 389 & 48 & $<.02$ \\
\hline
\end{tabular}


Table 2. Water-quality data collected from wells in Harrison County, Mississippi, during 1993--Continued

\begin{tabular}{|c|c|c|c|c|c|c|c|c|c|c|c|}
\hline $\begin{array}{l}\text { Site } \\
\text { number }\end{array}$ & $\begin{array}{l}\text { Local } \\
\text { number }\end{array}$ & $\begin{array}{l}\text { Geologic } \\
\text { unit } \\
\text { code }\end{array}$ & Date & $\begin{array}{r}\mathrm{Sp} \\
\text { condi } \\
(\mu \mathrm{S} \\
\text { field } \\
\end{array}$ & $\begin{array}{l}\text { ic } \\
\text { ance } \\
\text { n) } \\
\text { lab } \\
\end{array}$ & field & lab & $\begin{array}{c}\text { Color } \\
\text { (platinum- } \\
\text { cobalt } \\
\text { units) } \\
\end{array}$ & $\begin{array}{c}\mathrm{ROE} \\
(\mathrm{mg} / \mathrm{L})\end{array}$ & $\begin{array}{r}\text { Chloride } \\
\text { (mg/L ) } \\
r\end{array}$ & $\begin{array}{c}\text { Nitrite } \\
\text { plus } \\
\text { nitrate, as } \mathrm{N} \\
(\mathrm{mg} / \mathrm{L}) \\
\end{array}$ \\
\hline 136 & O6 & PCGL & June 30 & 433 & 449 & 8.4 & 8.6 & $<5$ & 284 & 19 & 0.03 \\
\hline 137 & O8 & GRMF & June 29 & 195 & 198 & 8.1 & 7.9 & $<5$ & 168 & 3.0 & $<.02$ \\
\hline 138 & 011 & GRMF & June 29 & 189 & 190 & 7.9 & 7.7 & $<5$ & 163 & 3.0 & $<.02$ \\
\hline 139 & O123 & PCGL & June 29 & 271 & 272 & 7.4 & 7.8 & 20 & 202 & 3.1 & $<.02$ \\
\hline 140 & O125 & HBRG & July 7 & 375 & 374 & 9.0 & 8.7 & $<5$ & 238 & 17 & $<.02$ \\
\hline 141 & $\mathrm{O} 251$ & PCGL & June 29 & 239 & 242 & 8.4 & 8.4 & $<5$ & 184 & 3.3 & $<.02$ \\
\hline 142 & O268 & PCGL & June 29 & 257 & 260 & 8.1 & 8.0 & $<5$ & 196 & 4.1 & $<.02$ \\
\hline 143 & O296 & PCGL & June 29 & 247 & 249 & 8.3 & 8.0 & $<5$ & 183 & 3.4 & $<.02$ \\
\hline 144 & O307 & HBRG & June 29 & 426 & 434 & 8.6 & 8.8 & $<5$ & 265 & 34 & $<.02$ \\
\hline 145 & O309 & PCGL & July 8 & 531 & 525 & 9.0 & 8.7 & 10 & 333 & 21 & $<.02$ \\
\hline
\end{tabular}




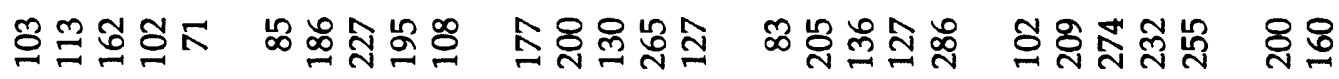

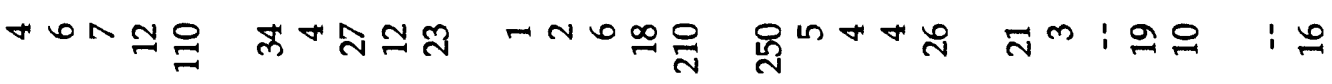

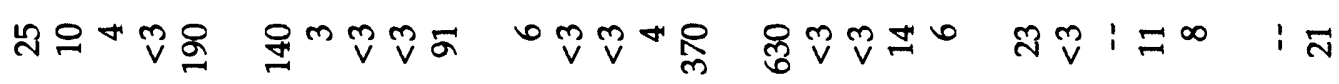

원도

우요 $\rightarrow$

$00 \sin ^{-1}$

유표유요 : กั่ง

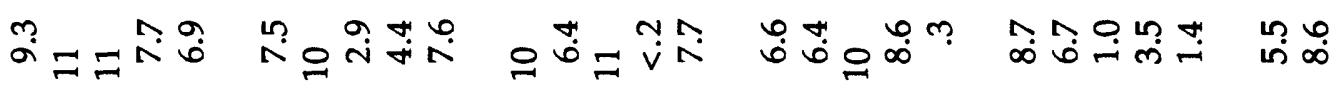

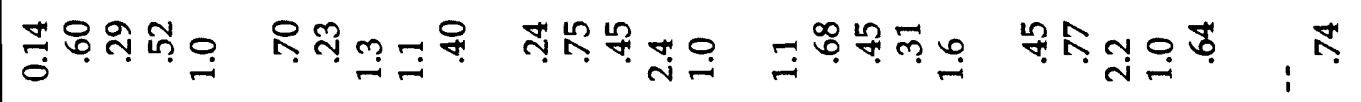

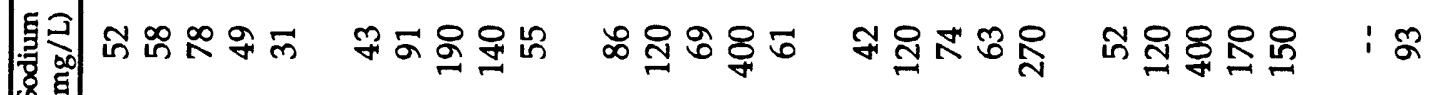
广遂

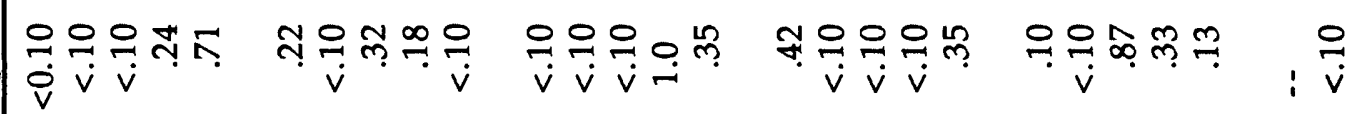

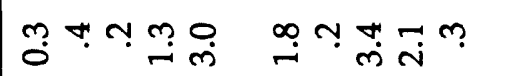

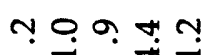

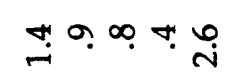

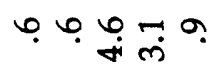

กับ

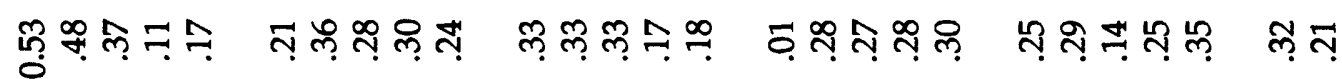

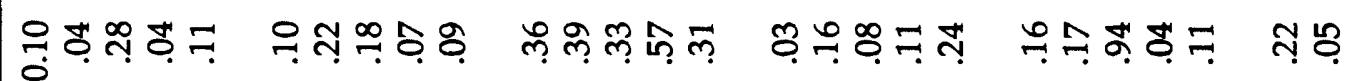

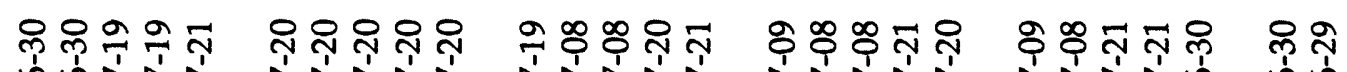

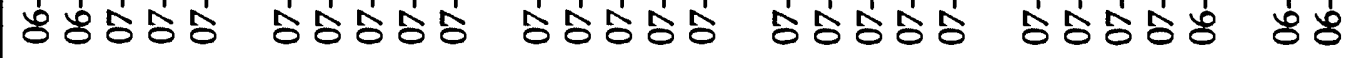

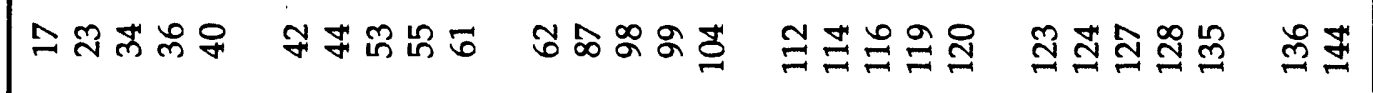




\section{Table 4. Summary of water-quality data collected from wells in Harrison County, Mississippi, during 1993}

$[\mu \mathrm{S} / \mathrm{cm}$, microsiemens per centimeter at 25 degrees Celsius; ROE, residue on evaporation at 180 degrees Celsius; mg/L, milligrams per liter; N, nitrogen; <, less than.

All constituents dissolved]

Depth, in feet

Specific conductance, field $(\mu \mathrm{S} / \mathrm{cm})$

Specific conductance, lab $(\mu \mathrm{S} / \mathrm{cm})$

$\mathrm{pH}$, field

$\mathrm{pH}$, lab

Color (platinum-cobalt units)

$\mathrm{ROE}(\mathrm{mg} / \mathrm{L})$

Chloride (mg/L)

Nitrite plus nitrate, as $\mathrm{N}(\mathrm{mg} / \mathrm{L})$

\section{Citronelle Formation}

(2 samples)

Minimum

80

23

21

5.5

6.1

$<5$

37

3.1

$<0.02$

$\begin{array}{cc}\text { Median } & \text { Maximum } \\ 115 & 150 \\ 192 & 360 \\ 188 & 354 \\ 7.0 & 8.5 \\ 7.3 & 8.5 \\ <5 & <5 \\ 136 & 236 \\ 6.4 & 9.8 \\ <0.02 & <0.02\end{array}$

Graham Ferry Formation

(55 samples)

Depth, in feet Minimum

Specific conductance, field $(\mu \mathrm{S} / \mathrm{cm})$

Specific conductance, lab $(\mu \mathrm{S} / \mathrm{cm})$

$\mathrm{pH}$, field

$\mathrm{pH}$, lab

Color (platinum-cobalt units)

ROE (mg/L)

Chloride (mg/L)

Nitrite plus nitrate, as $\mathrm{N}(\mathrm{mg} / \mathrm{L})$

Depth, in feet 74

13

15

Median

420

189

190

7.4

7.6

$<5$

157

3.5

$<0.02$
Maximum

700

434

429

9.2

8.9

120

296

12

0.85

\section{Pascagoula Formation}

(72 samples)

Specific conductance, field $(\mu \mathrm{S} / \mathrm{cm})$

$\underset{476}{\text { Minimum }}$

Specific conductance, lab $(\mu \mathrm{S} / \mathrm{cm})$

111

112

$\mathrm{pH}$, field

$\mathrm{pH}, \mathrm{lab}$

7.1

Color (platinum-cobalt units)

$<5$

$\mathrm{ROE}$ (mg/L)

129

Chloride (mg/L)

2.3

$<0.02$

Median
842
342
344
8.9
8.6
$<5$
220
4.2
$<0.02$

Maximum

1,320

2,000

2,020

Nitrite plus nitrate, as $\mathrm{N}(\mathrm{mg} / \mathrm{L})$

\section{3}

9.0

60

1,120

470

0.14 
Table 4. Summary of water-quality data collected from wells in Harrison County, Mississippi, during 1993--Continued

\begin{tabular}{|c|c|c|c|}
\hline \multicolumn{4}{|c|}{$\begin{array}{l}\text { Hattiesburg Formation } \\
\text { (14 samples) }\end{array}$} \\
\hline & Minimum & Median & Maximum \\
\hline Depth, in feet & 1,400 & 1,675 & 1,800 \\
\hline Specific conductance, field $(\mu \mathrm{S} / \mathrm{cm})$ & 230 & 400 & 1,240 \\
\hline Specific conductance, lab $(\mu \mathrm{S} / \mathrm{cm})$ & 225 & 372 & 1,260 \\
\hline $\mathrm{pH}$, field & 8.1 & 8.8 & 9.2 \\
\hline $\mathrm{pH}, \mathrm{lab}$ & 8.1 & 8.5 & 9.0 \\
\hline Color (platinum-cobalt units) & $<5$ & $<5$ & 20 \\
\hline $\mathrm{ROE}(\mathrm{mg} / \mathrm{L})$ & 150 & 246 & 711 \\
\hline Chloride $(\mathrm{mg} / \mathrm{L})$ & 1.9 & 11 & 230 \\
\hline Nitrite plus nitrate, as $\mathrm{N}(\mathrm{mg} / \mathrm{L})$ & $<0.02$ & $<0.02$ & 0.03 \\
\hline \multicolumn{4}{|c|}{$\begin{array}{c}\text { Catahoula Sandstone } \\
\text { (2 samples) }\end{array}$} \\
\hline & Minimum & Median & Maximum \\
\hline Depth, in feet & 2,400 & 2,405 & 2,410 \\
\hline Specific conductance, field $(\mu S / \mathrm{cm})$ & 590 & 860 & 1,130 \\
\hline Specific conductance, lab $(\mu \mathrm{S} / \mathrm{cm})$ & 592 & 851 & 1,110 \\
\hline $\mathrm{pH}$, field & 8.9 & 8.9 & 9.0 \\
\hline $\mathrm{pH}, \mathrm{lab}$ & 8.5 & 8.6 & 8.7 \\
\hline Color (platinum-cobalt units) & 10 & 10 & 10 \\
\hline $\mathrm{ROE}(\mathrm{mg} / \mathrm{L})$ & 339 & 488 & 638 \\
\hline Chloride $(\mathrm{mg} / \mathrm{L})$ & 73 & 146 & 220 \\
\hline Nitrite plus nitrate, as $\mathrm{N}(\mathrm{mg} / \mathrm{L})$ & $<0.02$ & $<0.02$ & $<0.02$ \\
\hline \multicolumn{4}{|c|}{$\begin{array}{l}\text { All geologic units } \\
\text { (145 samples) }\end{array}$} \\
\hline & Minimum & Median & Maximum \\
\hline Depth, in feet & 74 & 746 & 2,410 \\
\hline Specific conductance, field $(\mu \mathrm{S} / \mathrm{cm})$ & 13 & 280 & 2,000 \\
\hline Specific conductance, lab $(\mu \mathrm{S} / \mathrm{cm})$ & 15 & 273 & 2,020 \\
\hline $\mathrm{pH}$, field & 5.2 & 8.6 & 9.3 \\
\hline $\mathrm{pH}, \mathrm{lab}$ & 5.9 & 8.4 & 9.0 \\
\hline Color (platinum-cobalt units) & $<5$ & $<5$ & 120 \\
\hline $\mathrm{ROE}(\mathrm{mg} / \mathrm{L})$ & 20 & 199 & 1,120 \\
\hline Chloride $(\mathrm{mg} / \mathrm{L})$ & 1.9 & 3.7 & 470 \\
\hline Nitrite plus nitrate, as $N(\mathrm{mg} / \mathrm{L})$ & $<0.02$ & $<0.02$ & 0.85 \\
\hline
\end{tabular}

